BNL -52.336

DE92 019052

Physics

\title{
The Foxhole Accelerating Structure
}

R. C. Fernow and J. Claus

July 17,1992

Brookhaven National Laboratory

Associated Universitites, Inc.

Upton. Long Island, New York 11973

Under Contract No. DE-AC02-76CH00016 with the WITED STATES DEPARTMENT OF ENERGY

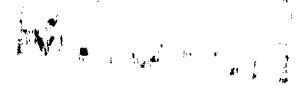




\section{THE FOXHOLE ACCELERATING STRUCTURE}

R.C. Fernow \& J. Claus

Brookhaven National Laboratory

11 May 1992

This report examines some properties of a new type of open accelerating structure. It consists of a series of rectangular cavities, which we call foxholes, joined by a beam channel. The power for accelerating the particles comes from an external radiation source and enters the cavities through their open upper surfaces. Analytic and computer calculations are presentad showing that the foxhole is a suitable structure for acoelerating relativistic electrons. 
CONTENTS

1. Introduction 1

2. Comparison with conventional linacs 1

3. General design criteria

4. Waveguide properties 5

5. Standing wave properties

6. Reflection coefficient 12

7. Wavelength response 15

8. RF cavity properties 18

9. Dimensional tolerances 22

10. Time averaged forces 24

11. MAFIA model 27

12. Slotted channel structure 30

$\begin{array}{ll}\text { 13. Buried channel structure } & 37\end{array}$

14. Design dimensions 43

15. Conclusions 44

Notes and references 45 


\section{Introduction}

I" is widely believed that the only practical means of pursuing accelerator-based particle physics after the SSC is through the construction of electron-positron linear colliders[1]. Cost optimization studies of these devices have indicated that the operating wavelength should be significantly shorter than the $10 \mathrm{~cm}$ used in the SLC, the first operating linear collider[2-3]. In addition, it has been proposed to investigate using the high peak power from lasers as the power source for the accelerator[4]. However, since the dimensions of the accelerating cavities scale with the wavelength, it becomes increasingly more difficult to construct iris-loaded, cylindrical cavities of the conventional type. This has lead to the proposal for an open, planar type of geometry for the accelerator cavities[5]. One of these open structures called the foxhole was first proposed by Claus[6] in 1986 .

In this report we continue the study of foxhole structures as accelerating cavities. Simple analytic and computer models are used to elucidate the expected accelerating gradient, coupling strength to the external field, and the dependence of these quantities on the dimensions of the cavity and the transverse position of the beam.

The results are somewhat prejudiced by the desire to design a structure suitable for $\lambda \approx 10 \mu \mathrm{m}$ that can be fabricated by methods that are conventional in the semiconductor industry. Foxholes deeper than $\frac{1}{2} \lambda$ and "buried beam channels" may be difficult to make at $\lambda=10 \mathrm{\mu m}$. Such restrictions may be less severe at longer wavelengths, e.g. $\lambda \approx 1 \mathrm{~mm}$. There is evidence that deeper foxholes and buried channels would be advantageous.

\section{Comparison with conventional 1 inacs}

Both the foxhole structure, shown in Fig. 1, and the conventional. electron linac consist of chains of resonating cavities aligned along the axis of the beam path. The transverse walls between adjoining cavities have holes for beam passage. The excitation methods however are quite different. In the conventional linac the power propagates from end to end along the system axis, passing from cavity to cavity via the beam passage holes. Each section of many cells has special couplers to guide the excitation power, which is generated off-axis, onto and away from the system axis. The cavities of the foxhole structure on the other hand are individually and independently coupled to a common power source, nore or less in the spirit of a sloan-luawence structure.

The acceleration process is the same in the two structures. The cavities carry an axial electric field in the vicinity of the beam 
FOXHOLE STRUCTURE / 2

path. The phase difference $\Delta \phi$ between successive cavities must match the propagation time $\Delta t$ of the particles

$$
\Delta \phi=\omega \Delta t=\frac{\omega L}{\beta C}
$$

where $\omega$ is the radian frequency, $L$ is the center to center distance between successive resonators, and $B c$ is the velocity of the particles. The tolerance on the average phase delay per cell, averaged over many cells, is very small because the phase of the

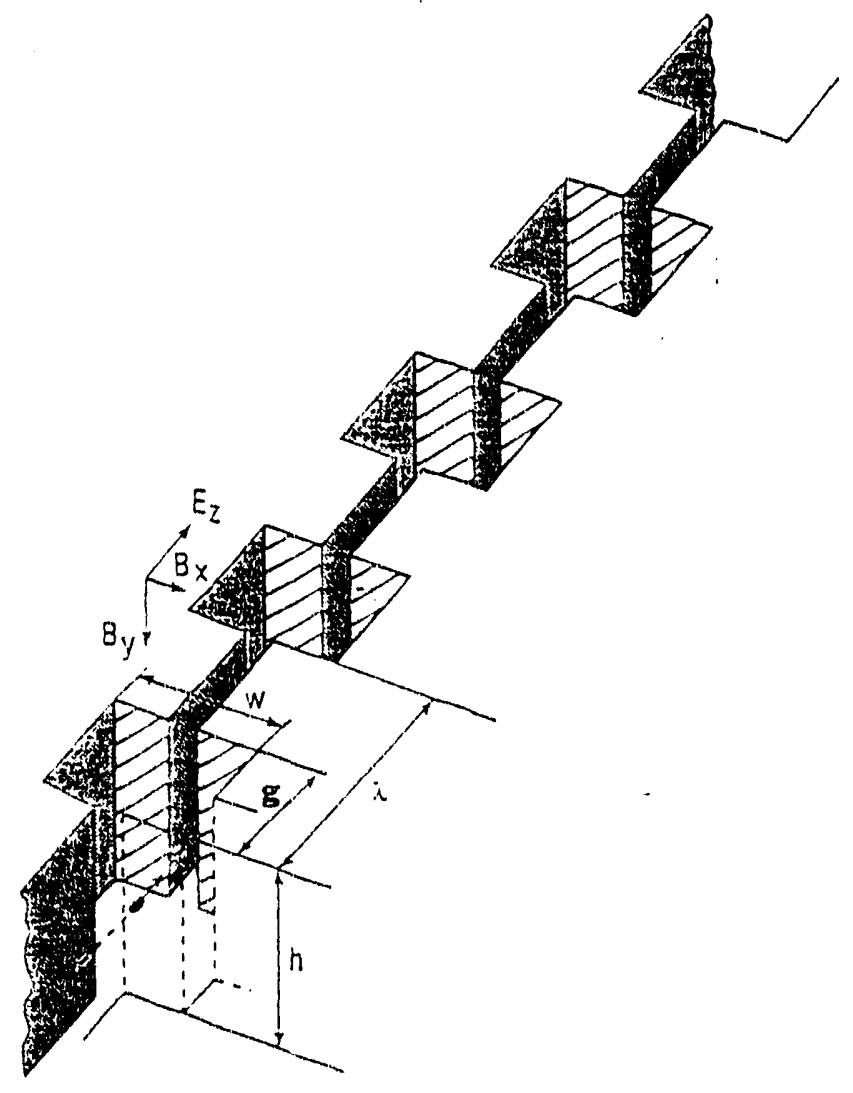

Fig. 1 Schematic diagram of a foxhole accelerating structure.

accelerating field seen by particles in the $n^{\text {th }}$ cell is

$$
\phi=\phi_{0}+\sum_{k=1}^{a} \Delta \phi_{k}
$$

This condition is a major restriction in the conventional linac, where each resonator is driven by the previous one via the coupling 
hole. Meeting the criterion requires very tight control over the resonant frequencies of the individual resonators, their axial. locations, and the dimensions of the coupling holes. However, the phase condition is relatively easy to satisfy in the foxhole structure. All the resonators are driven in parallel from a common power source that provides a common phase reference. The only critical parameter is the axial location of the centers of the resonators. The foxhole structure requires higher peak power than a conventional structure for the same frequency and accelerating gradient because all its resonators in a given section of accelerator are filled with energy simultaneously. The power must be provided during a time interval sufficiently long to guarantee that the first particle to enter and the last particle to leave the structure receive the same acceleration. In the case of single bunch acceleration the power must be provided for a time interval equal to a filling time plus the electron transit time plus one period. However, if the whole accelerator is subdivided into $n$ sections, the peak power per section becomes $1 / n$ of what it would be with no subdivision. In this case the pulse length scales like $1 / n$ of the total electron transit time until the pulse length approaches its limiting value of a fill time plus a period.

The $Q$ 's of the resonators do not have to be particularly high (1020 would be good enough) and therefore the filling time in terms of RF cycles can be small. The resonators of a conventional linac must have very high $Q^{\prime}$ s or else there will be few cells per section and many feed points. The signal velocity along the system axis tends to be quite low and therefore the filling time is large. As a result less power has to we supplied during a longer time than in

the foxhole arrangement. However, the higher the operating frequency, the lower the $Q$ of the resonators, and the more the conventional structure resembles the foxhole in tolerances and power requirements. We conclude that for very high frequencies (e.g. $\lambda<1 \mathrm{~cm}$ ) foxholes may be the way to go.

\section{General design criteria}

Foxhole structures can be designed at many levels of complexity. One goal may be to increase the ratio between the average accelerating gradient and the peak field on the surfaces of the resonator walls. Another goal is to achieve maximum efficiency. At this point in these studies, however, it seems prudent to forego sophistication in the interest of simplicity of fabrication and analysis. It seems unlikely that sophistication will increase the effective accelerating gradient by more than a factor of 2 above that in the simplest possible structures. Therefore in this section we discuss the general design parameters for a simple foxhole structure suitable for accelerating $50 \mathrm{MeV}$ electrons and for coupling to a $10 \mu \mathrm{m}$ power source $\left(\mathrm{CO}_{2}\right.$ laser).

A cection throllgh the midplane of the proposed structure is shown 
in Fig.1. We choose to have one resonator per wavelength. The resonator center to center distance $\mathrm{L}$ is

$$
L=\beta \lambda \sim\left(1-10^{-1}\right) \lambda
$$

where $\lambda_{0}$ is the free space wavelength. The incident radiation sees the resonators as sections of rectangular waveguide with cross section $w$ by $g$. We choose the accelerating gap $g \approx L / 2$. The width $w$ and height $h$ of the resonator are chosen to match the operating frequency. The beam moves from resonator to resonator via a slotted or buried channel with length $L-g$. The channels must have sufficient cross section to allow the beam to pass, but no more. The smaller this cross section the weaker the electromagnetic intercavity coupling. The cut off frequency of the channels, considered as waveguides, should be above the operating frequency.

The structure is excited by electromagnetic radiation that impacts perpendicularly onto the top surface and into the sections of waveguide. The electric component of the incident wave is directed along the electron beam. The structure resembles an Alvarez linac because it may be regarded as a collection of drift tubes (the channels or tunnels) that shield the electrons from the incident radiation field whenever that field is decelerating.

This shielding effect alone is not sufficient to make a useful accelerating structure because it leaves the traveling wave nature of the incoming radiation undisturbed. As we show later, there is in the waveguide a magnetic field component of the travelling wave that is in phase with the accelerating electric field, perpendicular to it and to the direction of propagation. Electrons travelling along the beam axis are therefore not on! accelerated longitudinally, but also transversely along the waveguide axis with nearly equal force. This transverse acceleration deflects the electrons off the axis and causes energy loss due to their emission of synchrotron radiation.

The problem with the deflecting magnetic field component can be solved by converting the travelling wave to a standing wave. The simplest way to accomplish this is to provide each resonator with a metallic floor at a point an odd number of quarter wavelengths below the particle beam axis. The incident radiation and the reflected wave combine to form a standing wave inside the resonator. As we show later the electric and magnetic field components are shifted relative to each other by a quarter wavelength along the waveguide direction and by a quarter period in time. As a result particles passing through at places where and at times when the electric field is maximum will feel little magnetic field. Unless the characteristic impedance of the guide is equal to that of free space the reflected radiation will be partially reflected again at the input end and the cavity will "fill" similar to a conventional one. 
It appears that a buried channel structure would have to be constructed from two aligned half-structures. However, the operation of the accelerating structure is not seriously compromised because, as we will see later, in the accelerating node no surface currents cross the symmetry plane of the structure.

At a later stage one might $c$ snsider converting this structure into an $R F$ quadrupole by modulating the channel cross section with axial position as shown in Fig. 2. This modulation introduces an alternating gradient focussing effect on the betatron motion. Another later refinement might be to replace the rectangular cavities with some other geometry (e.g. elliptical), if that offers an advantage for some particular problem.

We should point out that the following analysis only considers the properties of isolated foxholes. Surface currents must exist in the metallic parts of the upper plate of the structure. If the currents originating in a given foxhole have not been significantly attenuated by the time they reach a neighboring cavity, the structure will behave like a system of coupled resonators. This coupling has been assumed to be small in this report.

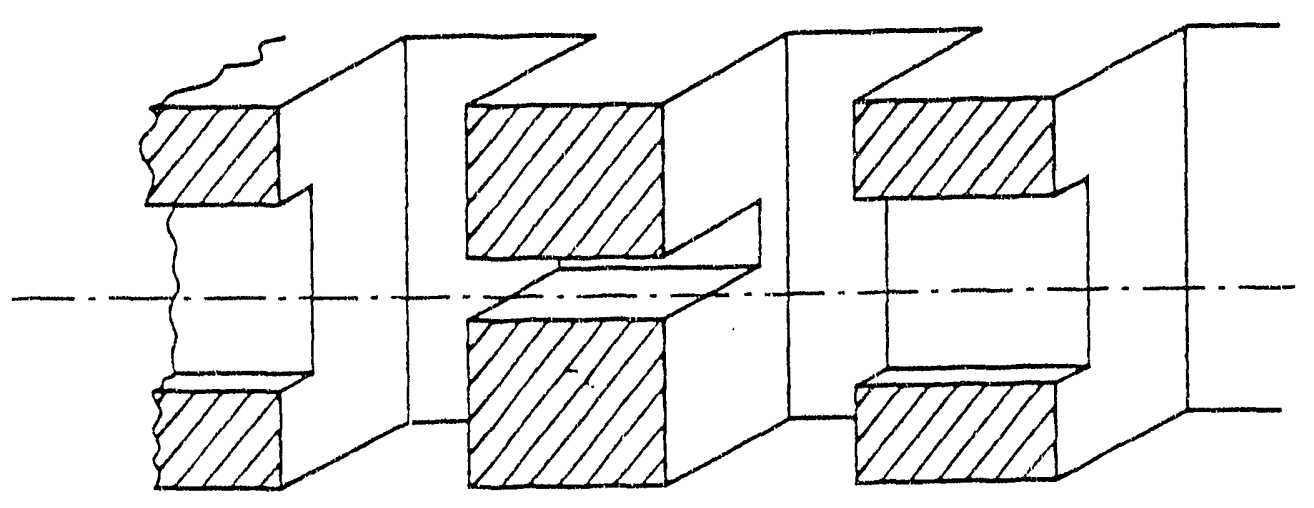

Fig. 2 Schematic diagram showing a vertical slice through an $R F$ quadrupole foxhole structure.

\section{Waveguide properties}

We now consider the form of the electromagnetic fields that exist inside the foxhole cavity. Unfortunately, it is probably not possible to derive an analytical solution of Maxwell's equations with the 3-dimensional boundary corresponding to a foxhole structure. Thus, in order to obtain accurate predictions of the coupling of the external radiation to the structure, it will 
FOXHOLE STRUCTURE / 6

eventually be necessary to use a 3-D numerical code that can accomodate arbitrary surface boundaries and true radiation boundary conditions at infinity. Until such a cods is available, we can only consider approximate solutions for the fields. We expect that these solutions give an adequate representation of the fields deep inside the cavity, but they will clearly be suspect in the region near the upper surface.

Far above the cavity the field consists of a plane wave incident downward and normal to the top surface of the structure, as shown in Fig. 3. We adopt a right-handed coordinate system, where the electron beam axis is along $z$ and the incident radiation is along $(-y)$. The $E$ field component is responsible for accelerating the pa ticles. The width of the guide is w and the accelerating gap is g. The incident wave is polarized with its $E$ vector along $z$. For the present discussion assume thet each of the rectangular cavities seen by the incident radiation extend infinitely far in the $-y$ direction. It is reasonable to assume that after a complicated transition region near the upper surface, the form of the waves inside the cavity must correspond to the solution for an electromagnetic wave in a matallic waveguide. Thus we want to examine the properties of $\mathrm{TE}$ travelling waves in the foxholes.
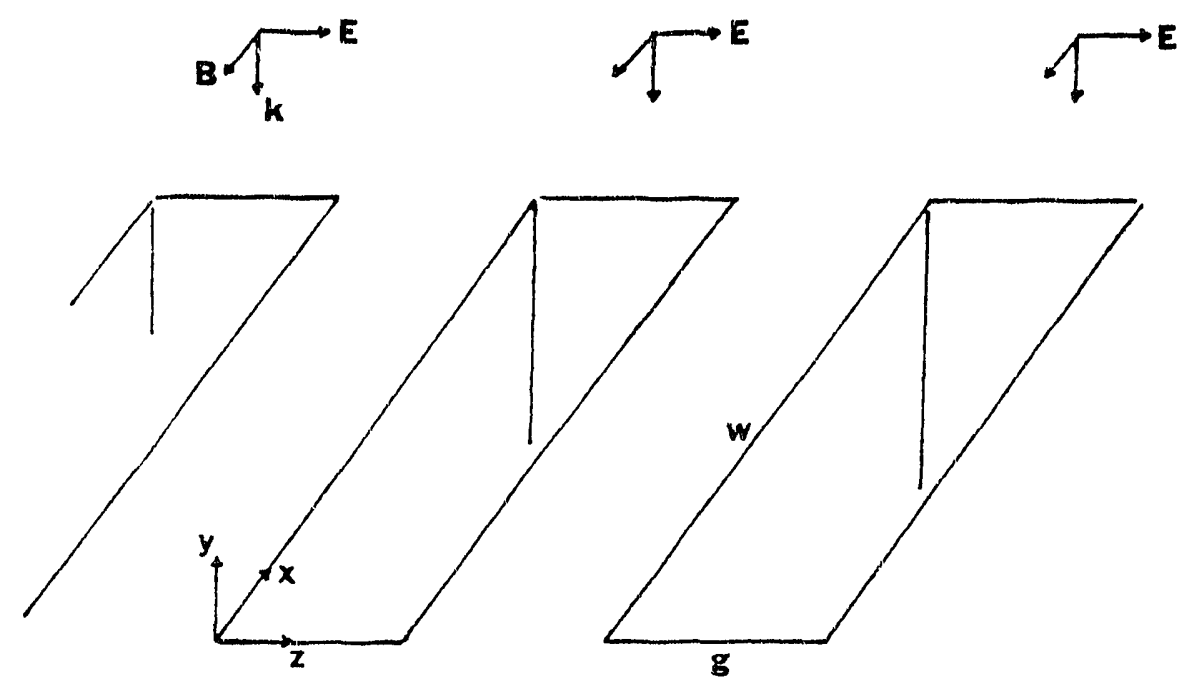

Fig. 3 Plane electromagnetic wave incident on the upper surface of a foxhole structure.

For a TE mode the only longitudinal field component in the 
waveguide is $B_{y}$, which can be written

$$
B_{y}=B_{0} \cos \left(\frac{m \pi x}{w}\right) \cos \left(\frac{p \pi z}{g}\right) e^{-i\left(k_{g} y+\omega t\right)}
$$

where $m$ and $p$ are integers and $k$ is the wavenumber inside the guide. In order to satisfy Maxwell's equations the wavenumber $k_{g}$ has to satisfy the eigenvalue relation

$$
\mu_{0} e_{0} \omega^{2}-k_{g}^{2}=\pi^{2}\left(\frac{m^{2}}{w^{2}}+\frac{p^{2}}{g^{2}}\right)=\gamma_{m p}^{2}
$$

For an accelerating cavity it is desirable to look for solutions that have a constant value of $E$, along $z$ and no other components of the electric field. In addition we restrict ourselves to the simplest possible behavior for the $x$ dependence of $E_{q}$, namely one half-wavelength along the $x$ direction. Thus we now confine the discussion to solutions with $\mathrm{m}=1$ and $\mathrm{p}=0$. We assume that all the field components have an $\exp \{-i \omega t\}$ time dependence.

The transverse travelling wave components in a waveguide can be determined from the longitudinal component[7]. The non-vanishing transverse components are

$$
\begin{aligned}
E_{z} & =\frac{i \omega \partial_{x} B_{y}}{\gamma_{10}^{2}} \\
& =\frac{-i \omega \pi}{\gamma_{10}^{2} \omega} B_{0} \sin \left(\frac{\pi x}{\omega}\right) e^{-1\left(k_{g} y+\omega t\right)}
\end{aligned}
$$

and [ 8$]$

$$
\begin{aligned}
B_{x} & =\frac{-i k_{g} \partial_{x} B_{y}}{\gamma_{10}^{2}} \\
& =\frac{i k_{g}^{\pi}}{\gamma_{10}^{2} w} B_{0} \sin \left(\frac{\pi x}{w}\right) e^{-i\left(k_{g} y+\omega t\right)}
\end{aligned}
$$

Note that the travelling wave has the $B_{x_{x}}$ component in phase with $E_{g}$ and the $B_{7}$ component $90^{8}$ out of phase. In order for the $m=1$ and $p=0$ mode to be the lowest in the guide, we must have $w>g$.

The wavelength in the guide is related to $k_{g}$ through $k_{g}=2 \pi / \lambda_{g}$. 
FOXHOLE STRUCTURE / 8

The eigenvalue relation can be written in terms of $\lambda_{g}$ in the form

$$
\frac{1}{\lambda_{g}^{2}}+\frac{1}{4 w^{2}}=\frac{1}{\lambda^{2}}
$$

where $\lambda$ is the wavelength of the incident radiation in free space. This can be expressed conveniently as

$$
\frac{\lambda_{g}}{\lambda}=\frac{1}{\sqrt{1-\left(\frac{\lambda}{2 w}\right)^{2}}}
$$

We see that the wavelength $\lambda_{\text {g }}$ in the guide is longer than the wavelength $\lambda$ in free space. The waveguide is cut off for incident radiation with wavelengths longer than $2 \mathrm{w}$. Thus for a fixed wavelength of incident radiation, we must choose the dimensions of the cavities such that $w>\lambda / 2$.

The characteristic impedance $Z_{f}$ is an important parameter for describing the properties of a waveguide[9].

$$
\begin{aligned}
Z_{g} & =\frac{E_{t x}}{H_{t x}}=\frac{E_{z}}{H_{x}} \\
& =z_{0} \frac{\lambda_{g}}{\lambda}
\end{aligned}
$$

where $Z_{0}=377 \Omega$ is the impedance of free space. Note that, since $\lambda_{\text {g }}$ is larger than $\lambda$, the characteristic impedance of the waveguide is larger than the impedance of free space.

\section{5 standing wave properties}

In order to make an accelerating cavity we must now consider the effect of adding a metallic floor to the waveguide considered in the previous section. For convenience we translate the origin of the coordinate system to a lower corner of the cavity, as shown in Fig. 4 . 


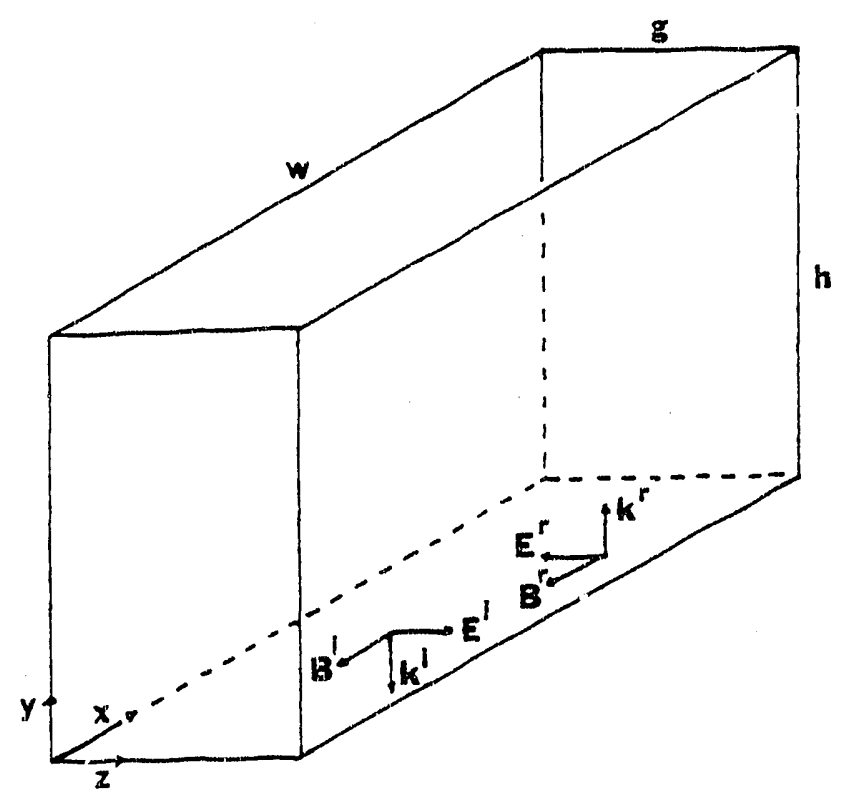

Fig. 4 The incident ( $i$ ) and reflected $(r)$ waves near the floor of the foxhole cavity.

After the travelling wave encounters the floor a second wave travelling in the ty direction exists inside the cavity. The boundary condition on the metal floor $(y=0)$ constrains $E_{\text {to }}$ to be zero for all $x$ and $t$. This requires that the amplitude $E_{,}{ }^{2}=-E_{0}{ }^{2}$, where the superscripts $i$ and $r$ refer to the incident and reflected wave. Then, since $E \times$ B gives the direction of propagation of the wave, $B_{1}$ does not change direction on reflection. The normal component of the magnetic vector $B$ must vanish on the metallic boundary, so $B_{7}^{*}=-B_{7}^{\prime}$. Adding the two travelling wave fields present inside the cavity gives a standing wave solution along $y$ :

$$
\begin{aligned}
& E_{z}=\frac{-2 \omega \pi B_{0}}{\gamma_{10}^{2}} \sin \left(\frac{\pi x}{w}\right) \sin \left(k_{g} y\right) e^{-1 \omega t} \\
& B_{x}=\frac{2 i k_{g} \pi B_{0}}{\gamma_{10}^{2} w} \sin \left(\frac{\pi x}{w}\right) \cos \left(k_{g} y\right) e^{-i \omega c} \\
& B_{y}=-2 i B_{0} \cos \left(\frac{\pi x}{w}\right) \sin \left(k_{g} y\right) e^{-1 \omega c}
\end{aligned}
$$

Vote that both components of the magnetic field are 30 out ot phase with respect, to the electric tield. In addition, since 3. aries like a cosine iunction of $y$ and $B_{7}$ varies like a cosiné :unction Dt $x$ wheroas

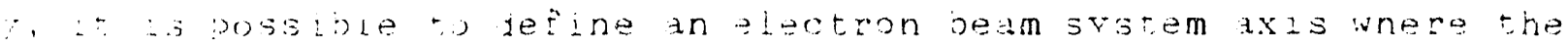


magnetic fielde seen by the p.rticles are small at all times, Thus it should be possible to use this structure to accelerate a particle bunch with a minimal amount of magnetic deflection.

The next question that we must address is how to roiate the wavelength $\lambda$, with the depth $h$ of the cavity. This ralation depends intimately on the nature of the fields in the vicinity of the open top of the cavity. Since we do not yet know the exact form for these fields, we are forred to maka assumptions about how the conditions there constrain the standing wave solution in the cavity. One thirg that we do know is that the plane wave field that exists far abnve the structure does not have a $B_{\text {g }}$ component, while the travelling and standing wave solutions inside the cavity must have such a component. It would thus seem plausible to watch these conditions at the open surface of the cavity by requiring that the standing wave have a zero in $B_{p}$ at that location. This would require that

$$
\begin{aligned}
k_{g} h & =n \pi \\
h & =n \frac{\lambda g}{2}
\end{aligned}
$$

in Eq. 11, where $n$ is an integer; we refer to these as interger mode solutions.

Consider a cross section of the foxhole structure shown in Fig. 5 a. The waves marked A represent three parts of the incident plane wave. At the same instant in time there must also be reflected wave. (B) so that the total electric field vanishes on the upper metallic surface. Let us consider solutions where the reflected wave (C) from the bottom of the cavity arrives at the upper boundary identical to the waves $B$. Because of the phase reversal in $E_{\text {g at }}$ the bottom of the cavity, this requires that the incident wave have the configuration $D$ just before and configuration $E$ just after striking the bottom surface. Note that on the boundary $y=h$ these 50 lutions also have $E_{:}=0$ and a finite, constant value of $H_{x}$, independent of $z$. A surface current density, shown in $F i g$. $5 \mathrm{~b}$, flows along $z$ on the horizontal sections and along $y$ on the vertical sections in the $f$ igure. The magnitude and phase of these currents are determined by the local value of $B_{\mathrm{r}}$. Thus according to $E q .13$ they have a sinusoidal dependence on $x$ across the foxhole. They are largest near the corners and zero at the midpoint of the 
vertical sections.
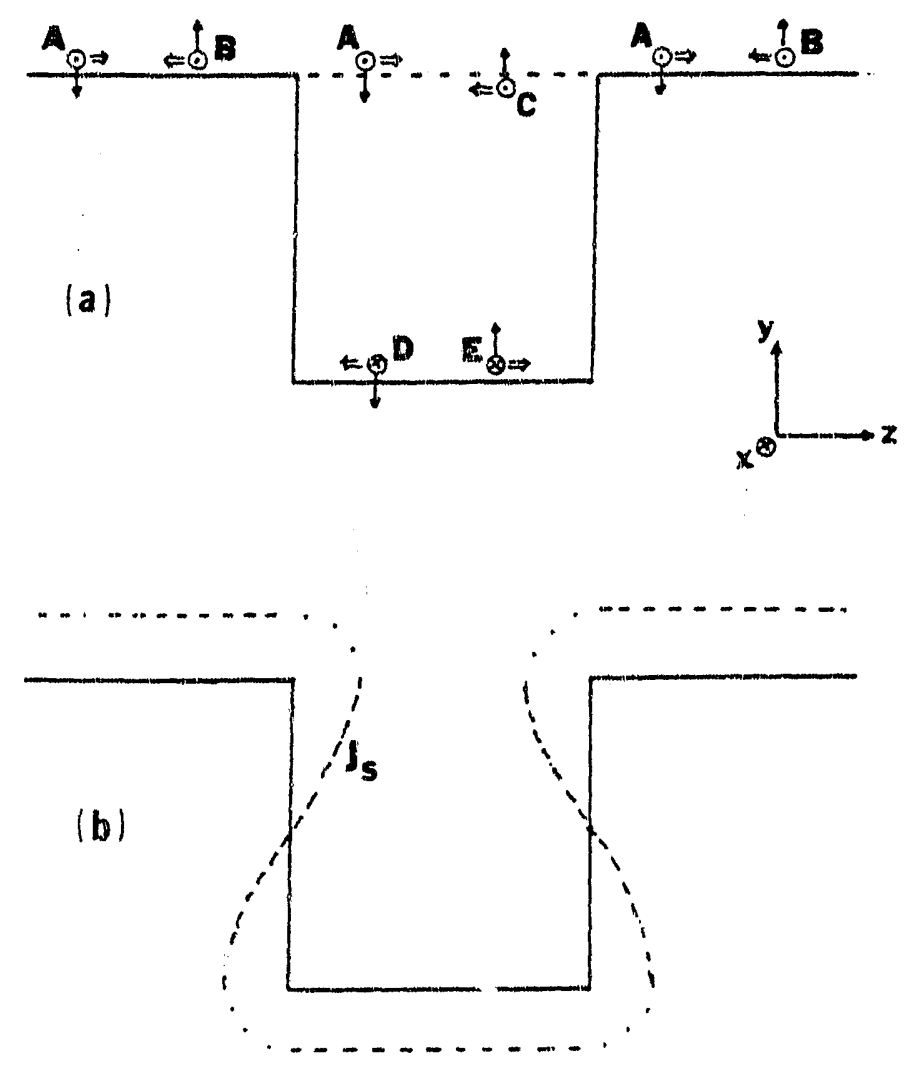

Fig. 5 (a) The incident plane wave $(A, D)$ and the reflected plane wave $(B, C, E)$ for an integer mode foxhole. Double arrows indicate the electric field. (b) The surface current density distribution on the edge of the foxhole. The magnitude and direction of the current is indicated by the distance of the curve from the surface.

Now if we take the real parts of the field components given in Eqs. 11 , substituta $\mathrm{Eq}, 12$ for $\mathrm{k}_{\mathrm{f}}$, and redefine the amplitude of $\mathrm{E}_{\mathrm{z}}$ to be $E_{A}$, the fielcs in the cavity can be written as

$$
\begin{aligned}
& E_{x}=E_{A} \sin \left(\frac{\pi x}{W}\right) \sin \left(\frac{n \pi y}{h}\right) \cos (\omega t) \\
& B_{x}=\frac{-n \lambda}{2 h} \frac{E_{A}}{C} \sin \left(\frac{\pi x}{\omega}\right) \cos \left(\frac{n \pi y}{h}\right) \sin (\omega t) \\
& B_{y}=\frac{\lambda}{2 W} \frac{E_{A}}{C} \cos \left(\frac{\pi x}{w}\right) \sin \left(\frac{n \pi y}{h}\right) \sin (\omega t)
\end{aligned}
$$

The equation for $B_{k}$ assumes we are treating the case with $\omega=c k$. 
FOXHOLE STRUCTURE / 12

If we substitute these components into the Maxwell equation

$$
\partial_{x} H_{y}-\partial_{y} H_{x}=\partial_{e} D_{x}
$$

we find that the resonance frequency of the cavity must be

$$
f_{x}=\frac{c}{2} \sqrt{-\frac{2}{w^{2}}+\frac{n^{2}}{h^{2}}}
$$

Fig. 6 shows the magnetic and electric field patterns for the fundamental $(n=1)$ integer mode field pattern.
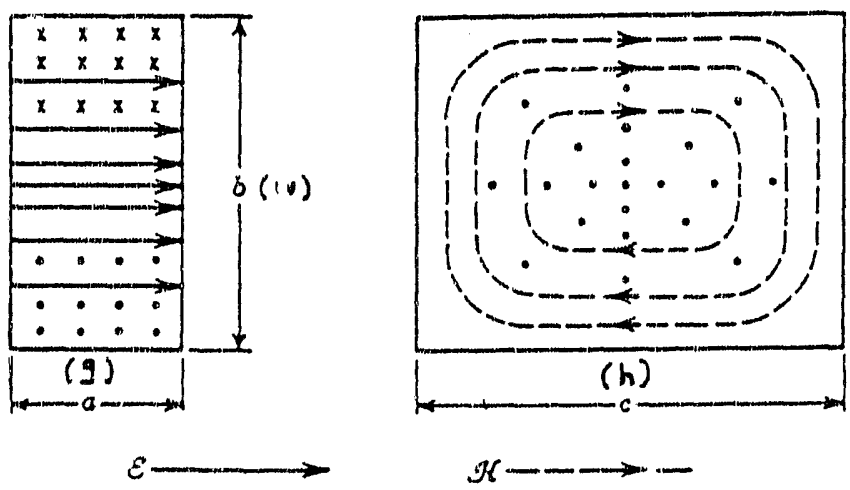

$\mathscr{K} \longrightarrow-\longrightarrow$

Fig. 6 The spatial pattern of the field components for the $n=1$ mode of the foxhole cavity [13].

\section{Reflection coefficient}

It is important to estimate the amplitude of the accelerating field in the foxhole for a given amplitude of electric field in the incident plane wave. We loosely refer to the ratio of these amplitudes as the "coupling strength" of the radiation. In order to do this let us first consider a wave travelling in the -y direction in a region with characteristic impedance $z_{1}$. The wave could be either a plane wave in free space or a TE waveguide mode. If this wave encounters a boundary to a region of space with characteristic impedance $Z_{2}$, it sfilts into a transmitted wave that continues on in the $-y$ direction and a reflected wave that heads back up along the $y$ direction. The relative amplitudes and phases of the incident, reflected, and transmitted waves are such that the boundary conditions with $z$, on one side and $Z$, on the other are satisfied. We define this boundary to be $y=0$ for convenience. 
The boundary conditions on the tangential components or the $E$ and $H$ vectors at $y=0$ and for any time require that

$$
\begin{aligned}
E_{z}^{i}(x, z)+E_{z}^{I}(x, z) & =E_{z}^{c}(x, z) \\
-H_{x}^{L}(x, z)+H_{x}^{z}(x, z) & =-H_{x}^{C}(x, z)
\end{aligned}
$$

where the superscript ( $t$ ) refers to the transmitied wave. The transverse components of the fields on each side of the boundary have the same, factorable dependence on $x$ and $z$. This allows us to rewrite the boundary conditions in terms of the amplitudes of the waves as

$$
\begin{gathered}
E_{0}^{i}+E_{0}^{I}=E_{0}^{t} \\
-\frac{E_{0}^{I}}{Z_{1}}+\frac{E_{0}^{I}}{Z_{2}}=-\frac{E_{0}^{t}}{Z_{2}}
\end{gathered}
$$

Define the reflection coefficient $r$ and the transmission coefficient to be the ratio of amplitudes

$$
\begin{aligned}
& I=\frac{E_{0}^{r}}{E_{0}^{i}} \\
& \tau=\frac{E_{0}^{t}}{E_{0}^{i}}
\end{aligned}
$$

so that

$$
\begin{aligned}
& E_{0}^{I}=I E_{0}^{i} \\
& E_{0}^{T}=I B_{0}^{+}=(1+I) E_{0}^{1}
\end{aligned}
$$

Dividing Eqs. 17 by $E_{f}^{i}$ and solving for $r$, we find

$$
I=\frac{z_{2}-z_{1}}{z_{1}+z_{2}}
$$

\footnotetext{
We see that the reflection coefficient can be either positive or negative depending on the relative magnitudes of the impedances on the two sides of the boundary.

The energy flow across the boundary is determined rrom the poynting vector
} 
FOXHOLE STRUCTURE / 14

$$
\vec{P}=\vec{E} \times \vec{H}
$$

The fraction of the energy incident on the boundary that is reflected is given by the reflectivity

$$
\begin{aligned}
R & =\left|\frac{\vec{p}^{x} \cdot y}{\vec{P}^{I} \cdot y}\right| \\
& =I^{2}
\end{aligned}
$$

The fraction of the energy incident on the boundary that is transmitted is given by the transmissivity

$$
\begin{aligned}
T & =\left|\frac{\vec{p}^{t} \cdot y}{\vec{p}^{1} \cdot y}\right| \\
& =\frac{Z_{1}}{Z_{2}}(1+r)^{2}
\end{aligned}
$$

It is a simple exercise to show that $R+T=1$, demonstrating that these expressions satisfy conservation of energy.

Now consider the case of a wave from free space incident on the upper boundary of the foxhole cavity. Thus we have $z_{1}=z_{0}$ and $z_{2}=$ $Z_{g}$. Using Eq. 10 we find

$$
I=\frac{1-\sqrt{1-\left(\frac{\lambda}{2 w}\right)^{2}}}{1+\sqrt{1-\left(\frac{\lambda}{2 w}\right)^{2}}}=
$$

Note that in the limit that $w \gg \lambda, r \approx 0$ and all the incident energy is transmitted into the waveguide. When $w=\lambda / 2$ the waveguide is cut of,$r=1$, and we have total reflection.

Next we want to consider what happens when the wave reflected from the bottom of the foxhule returns to the upper boundary. Eq. 20 for the reflection coefficient is still valid. However, we have $z_{1}=z_{3}$

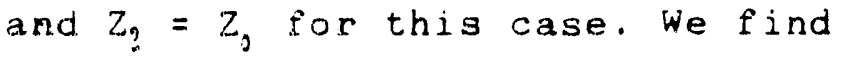

$$
r=-\vec{L}
$$

This has the same magnitude as the previous case, but has the opposite sign.

Table $i$ sives numerical examples for the dependence ot the quide wavelength, guide impedance and reflection coefficient on the width ot the foxhole. 


\begin{tabular}{|c|c|c|c|}
\hline & Cavity & properties & $\lambda=10.6$, \\
\hline$w$ & $\lambda_{8}$ & $z_{B}[\Omega]$ & $r$ \\
\hline 6 & 22.614 & 804 & 0.362 \\
\hline 7 & 16.227 & 577 & 0.210 \\
\hline 8 & 14.151 & 503 & 0.143 \\
\hline 9 & 13.115 & 466 & $0.100^{\circ}$ \\
\hline 10 & 12.500 & 445 & 0.082 \\
\hline 11 & 12.097 & 430 & 0.066 \\
\hline 12 & 11.815 & 420 & 0.054 \\
\hline 16 & 11.234 & 400 & 0.029 \\
\hline 20 & 10.993 & 391 & 0.018 \\
\hline
\end{tabular}

\section{Wavelength response}

We have seen that electromagnetic energy in the foxhole will be reflected back and forth between the upper and lower boundary surfaces. After a number of cycles the fields inside the foxhole cavity will approach an equilibriun value. Let us consider a cavity with reflection coefficient $r_{1}$ at the bottom surface and $r_{2}$ at the upper surface. Let $y$ be the distance measured up from the floor of the cavity. Assume that the initial wave is travelling in the $-y$

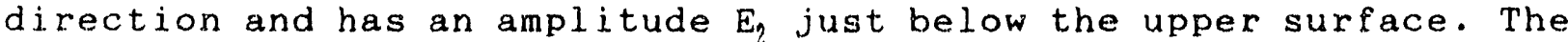
total field at any height $y$ in the cavity can be found by summing the contributions of all the reflected waves. We find

$$
\frac{E(y)}{E_{2}}=\frac{e^{i \alpha / 2}\left[e^{-i k_{g} y}+I_{1} e^{i k_{g} y}\right]}{1-I_{1} I_{2} e^{i \alpha}}
$$

where

$$
\alpha=2 k_{g} h
$$

If we now restrict ourselves to the case of a metallic floor, where 
FOXHOLE STRUCTURE, 16

$r_{1}=-1$, we obtain

$$
\frac{E(y)}{E_{2}}=\frac{-2 i e^{1 / 2} \sin \left(k_{q} y\right)}{1+I_{2} e^{1 /}}
$$

Define

$$
\begin{aligned}
\rho(y) & =\left|\frac{E_{z}(y)}{E_{2}}\right| \\
\tan \phi(y) & -\frac{\operatorname{Im}\left[E_{z}(y)\right]}{\operatorname{Re}\left[E_{z}(y)\right]}
\end{aligned}
$$

where $p(y)$ and $\Phi(y)$ are are the relative magnitude and phase of the total field at the height $y$. The reflection coefficient at the upper surface must be negative, as we mentioned in the previous section. Thus we have $r_{2}=-r$, where $r$ is given by Eq. 24. The magnitude can be written as

$$
p=\frac{2 \sin k_{g} y}{\sqrt{1-2 x \cos \alpha+y^{2}}}
$$

The phase relation can be written as

$$
\tan \Phi=-\frac{(1+r)}{(1-r)} \cot \frac{\alpha}{2}
$$

or equivalently as

$$
\Phi=\frac{3 \pi}{2}+k_{g} h+\tan ^{-1}\left[\frac{r \sin \alpha}{1-r \cos \alpha}\right]
$$

It is desirable to relate the field enhancement factor $p$ to the strength of the incident wave $E_{0}$. In order to do this we assume that the dominant spatial Fourier component of the actual field that exists just above the upper surface of the foxhole has an amplitude comparable in magnitude to that of the incident field. Then the amplitude of the field that gets into the foxhole is

$$
E_{2} \nsim \tau E_{0}
$$

and we can write the field at the height $y$ as

$$
E_{z}(y) \sim p(y) \tau E_{0}
$$

This shows that the resultant field depends on the resonant properties of the cavity through the factor $\rho$, on the coupling efficieny through the factor $\tau$, and on the strength of the incident field through the factor $E_{0}$. 
As an example consider an accelerating cavity where we fix the value of $y$ to be a quarter guide wavelength above the floor of the cavity, so that the electron beam sees the maximum accelerating field. We fix the height of the cavity to be a half guide wavelength, so that there is a zero in the electric field at the upper boundary. For this case the $r$ dependence in $E(y)$ coming from $\rho$ cancels that from $\tau$ and we find that $E(y)=2 E$, independent of the cavity dimensions. The phase is $90^{\circ}$ for this case. We can relate this discussion to that of the standing wave fields by noting that $E_{d}=E_{\eta}(h / 2)$. The field on the accelerator axis $E_{\text {is }}$ related to the field just inside the open surface of the foxhole $E_{2}$ by

$$
E_{A} \approx \frac{2 E_{2}}{1-I}
$$

Now with the cavity dimensions and $y$ fixed with values corresponding to the resonant frequency of the cavity, let us vary the wavelength of the incident radiation. The dependence of the field enhancement $\rho \tau$ and phase $\Phi$ on $\lambda$ is shown in Fig. 7 . In a later section we will use the typical resonance response shown in Fig. 7 to determine the dimensional tolerances required for building a foxhole accelerator.

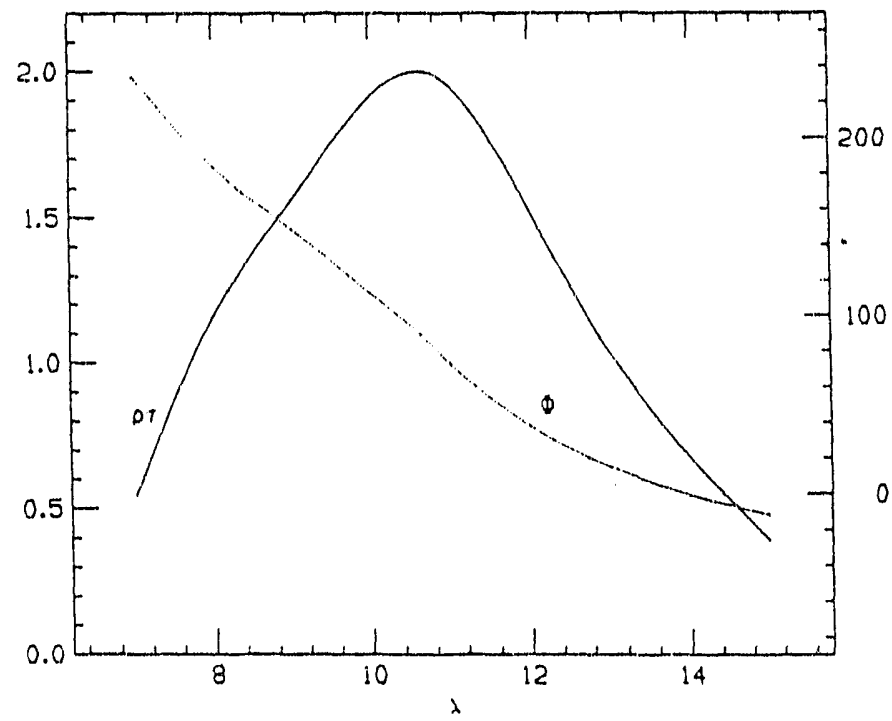

Fig. 7 The magnitude ( $\rho$, left scale) and phase ( $\Phi$, right scale) of the accelerating electric field in the foxhole as a function of the wavelength of the incident radiation. $w=8$ and $g=5.3$. 
FOXHOLE STRUCTURE / 18

\section{RF cavity properties}

Important radiofrequency (RF) properties of an accelerating cavity include the stored energy, power loss, quality (Q) factor, shunt impedance, and the fill time. The RF properties of the foxhole cavity are given in Table 2 for a number of different values of $w$.

\section{Stored energy}

The total stored energy in the foxhole can be approximated by integrating the energy density over the geometrical dimensions of the foxhole. The total stored energy in the cavity is

$$
U \sim \frac{1}{8} e_{0} E_{A}^{2} g w h
$$

\section{Power loss}

There are two main sources of power loss in a foxhole. First there is the Joule heating loss due to the finite conductivity of the metallic walls. The second and dominant loss is the escape of radiation through the upper, open boundary. Consider first the dissipative Joule losses. Surface currents flowing through the finite resistance of the cavity walls cause power losses from the electromagnetic field. These currents $\left(I_{S}\right)$ can be simply related to the value of the magnetic field parallel to the surface $\left(H_{s}\right)$ by using the integral form of Ampere's law.

$$
H_{S} w^{\prime}=I_{S}
$$

where $w^{\prime}$ is the width of the distribution of surface current. Eq. 13 shows that the lines of $H_{y}$ are circular around the beam axis and independent of $z$. Thus there are surface currents flowing back and forth between the beam entrance and exit walls of the cavity. These currents flow along the bottom and the two sides of the cavity.

The time-averaged power absorbed per unit surface area is [7]

$$
\frac{d P_{d}}{d A}=\frac{1}{20 \delta} H_{s}^{2}
$$

where $\sigma$ is the electrical conductivity of the metallic surface and $\delta$ is the skin depth, defined as 


$$
\delta=\sqrt{\frac{2}{\omega \mu \sigma}}
$$

Thus the total power loss can be written

$$
P_{d}=\frac{1}{2 \sigma \delta} \sum \int H_{s}^{2} d A
$$

where the sum is over the five metallic walls. After perfcrming the integrals, we find that the resistive power loss for the limiting case of no beam channel is

$$
P_{d}=\frac{1}{808}\left(\frac{E_{\lambda} \lambda}{Z_{0}}\right)^{2} F
$$

where the dimensionless factor $F$ is

$$
F=\left[\frac{g h}{w^{2}}+\frac{g w n^{2}}{2 h^{2}}+1 / 2\left(\frac{w n^{2}}{h}+\frac{h}{w}\right)\right]
$$

Alternatively, we can express this in the form

$$
P_{d}=\frac{1}{208}\left(\frac{E_{\lambda} w}{Z_{0}}\right)^{2} \frac{E}{\left[1+\left(\frac{n W}{h}\right)^{2}\right]}
$$

Now let us consider the power loss due to radiation. The time average power flowing into the foxhole just below the open upper surface of the cavity is

$$
P=1 / 2 \int \vec{E}_{z} \times \vec{H}_{x} d A
$$

where $\mathrm{dA}$ is an element of the cross sectional area of the cavity. Using the traveling wave form of the field components with an amplitude $E_{2}$ as given in the previous section, we find

$$
P=\frac{E_{2}^{2} g w}{4 Z_{g}}
$$

Then using Eq. 35 for $E_{2}$, we find that the total power flowing into 
FOXHOLE STRUCTURF / 20

the cavity is

$$
P=\frac{(1-I)^{2} E_{\lambda}^{2} g W}{16 Z_{g}}
$$

Some of the incoming power is dissipated due to ohmic losses in the cavity walls and to interaction of the fields with the electron beam. If we assume the beam loading is negligible, the power that is not lost in the walls must be radiated back out of the cavity under steady state conditions. Thus we can write the radiated power as

$$
P_{s}=P-P_{d}
$$

The numerical examples given in Table 2 show that the power loss will be dominated by the radiation losses through the open surface.

\section{Q factor}

The $Q$ of a cavity is often defined as

$$
Q=\frac{\omega U}{P}
$$

where $U$ is the total electromagnetic energy stored in the cavity and $P$ is the total power loss. In conventional cavities the major source of loss is due to the resistive dissipation of surface currents. For structures like the foxhole on the other hand, the major source of loss is due to radiation through the open boundary. Using Eq. 46 for the total power loss, we find

$$
Q=\frac{2 \pi n}{(1-I)^{2}}\left(\frac{\lambda_{g}}{\lambda}\right)^{2}
$$

Note that since $h$ is proportional to $\lambda_{f}$ the $Q$ increases rapidly as the depth of the foxhole is increased.

\section{Fill time}

The fill time of a cavity is defined to be the time for the electric field in the cavity to reach the fraction $(1-1 / e)$ of its final value. We can derive an expression for the fill time in terms of the reflection coefficient. We have given in Eq. 26 an expression for the field in the cavity after an infinite number of reflections. If instead we ask for the value of the field after a 
finite number $N$ of reflections, we find

$$
E_{A}^{N}=E_{A}^{\infty}\left(1-r^{N}\right)
$$

The fraction of the ultimate field strength achieved after $\mathrm{N}$ cycles is

$$
\xi-\frac{E_{A}^{N}}{E_{A}^{\infty}}=1-I^{N}
$$

Therefore, the number of cycles (or filling time) to achieve a given fraction $\xi$ of the ultimate field strength is

$$
N(\xi)=\frac{\ln (1-\xi)}{\ln (r)}
$$

If we let $\xi_{0}=1-1 / e$ represent the fraction of the asymptotic field reached after one fill time, the corresponding number of cycles according to Eq. 52 is $N_{0}=-1 / \ln (r)$. The fill time is just $N_{0}$ times the pericd of the oscillation $T$

$$
t_{p}=\frac{-T}{\ln (r)}
$$

\section{Shunt impedance}

The effective shunt impedance is defined as

$$
R_{s h}=\frac{V_{A}^{2}}{P_{1 s c}}
$$

where $V_{A}=E_{A} g$ is the voltage across the cavity at the location of the accelerated beam and $P_{i n c}$ is the total power incident on the top of the structure. We assume that the radiation is incident on the top of the structure with a line focus of width $\approx w$. In this case only half the incident power will be available above the foxhole opening. The power just inside the opening will be

$$
P_{2} \sim 1 / 2 T P_{1 n c}
$$

where $T$ is the transmissivity. Using Eq. 23 for $T$ and Eq. 35 for relating $E_{2}$ to $E_{A}$, we find 
FOXHOLE STRUCTURE / 22

$$
R_{\mathrm{Bh}}-8 z_{0} \frac{g}{V}\left(\frac{1+I}{1-I}\right)^{2}
$$

The shunt impedance per unit length is

$$
\begin{aligned}
I_{a b} & =\frac{R_{s b}}{2 g} \\
& =4 \frac{Z_{0}}{W}\left(\frac{1+I}{1-I}\right)^{2}
\end{aligned}
$$

\begin{tabular}{|c|c|c|c|c|c|c|}
\hline & $E_{A}=1$ & $\mathrm{~V} / \mathrm{m}$; & $\lambda=10$ & $\mu m$; & $g=\lambda / 2$ & \\
\hline w & $\mathrm{h}$ & $U$ & $P_{\text {rad }}$ & $P_{d}$ & $Q$ & $\mathrm{r}_{\mathrm{sh}}$ \\
\hline$\mu \mathrm{m}$ & $\mu \mathrm{m}$ & $\mathrm{pJ}$ & $\mathrm{kW}$ & W & $\mathrm{Eq} .49$ & $M \Omega / m$ \\
\hline 6 & 11.307 & 398 & 0.60 & 412 & 70.2 & 1144 \\
\hline 7 & 8.113 & 333 & 2.21 & 298 & 23.6 & 505 \\
\hline 8 & 7.076 & 332 & 3.59 & 277 & 15.3 & 336 \\
\hline 9 & 6.558 & 346 & 4.83 & 280 & 12.0 & 257 \\
\hline 10 & 6.250 & 366 & 5.99 & 292 & 10.4 & 210 \\
\hline 11 & 6.048 & 390 & 7.08 & 309 & 9.4 & 179 \\
\hline 12 & 5.907 & 416 & 8.13 & 328 & 8.7 & 156 \\
\hline 16 & 5.617 & 527 & 12.1 & 420 & 7.5 & 106 \\
\hline 20 & 5.497 & 645 & 15.8 & 520 & 7.0 & 81 \\
\hline
\end{tabular}

\section{Dilmensional tolerances}

The resonant wavelength of the cavity can be found using Eq. 15

$$
\frac{4}{\lambda^{2}}=\frac{1}{w^{2}}+\frac{n^{2}}{h^{2}}
$$

The variation in the cavity resonance wavelength due to errors in the cavity dimensions is given by 


$$
\delta \lambda=\left[\left(\frac{\partial \lambda}{\partial w} \delta w\right)^{2}+\left(\frac{\partial \lambda}{\partial h} \delta h\right)^{2}\right]^{1 / h}
$$

where $8 w$ and $8 \mathrm{~h}$ are the dimensional errors in $w$ and $h$ respectively. We can find the dependence of $\lambda$ on $w$ and $h$ by taking the differential of Eq. 58

$$
\frac{4}{\lambda^{3}} d \lambda=\frac{1}{w^{3}} d w+\frac{n^{2}}{h^{3}} d h
$$

Assume that the dimensional errors $\delta \mathrm{w}$ and $\delta \mathrm{h}$ are both determined to an accuracy $\delta x$. We find that the wavelength error is

$$
\delta \lambda=G \delta x
$$

where the cavity dimensions are contained in the dimensionless factor

$$
G=\frac{1}{4}\left(\frac{\lambda}{w}\right)^{3}\left[1+n^{4}\left(\frac{w}{h}\right)^{6}\right]^{1 / 2}
$$

For a given application there will be some acceptable frequency spread, which we call $\Delta f$, and a corresponding acceptable spread in wavelength $\Delta \lambda$. The acceptable dimensional tolerance $\Delta x$ is then given by Eq. 61. Two problems arise if the resonant frequency is not matched to that of the incident wave. The amplitude of the cavity field is reduced and its phase relative to the incident wave changes. If the error is due to an error in $h$, the electrons, which travel at a fixed depth in the cavity, will not be located at an extremum in the cavity standing wave.

The tolerance properties for an example foxhole cavity are shown in Table 3. We adopt as our wavelength criterion that the phase variation lie within $\pm 20^{\circ}$ of resonance in Fig. 7 . 
FOXHOLE STRUCTURE / 24

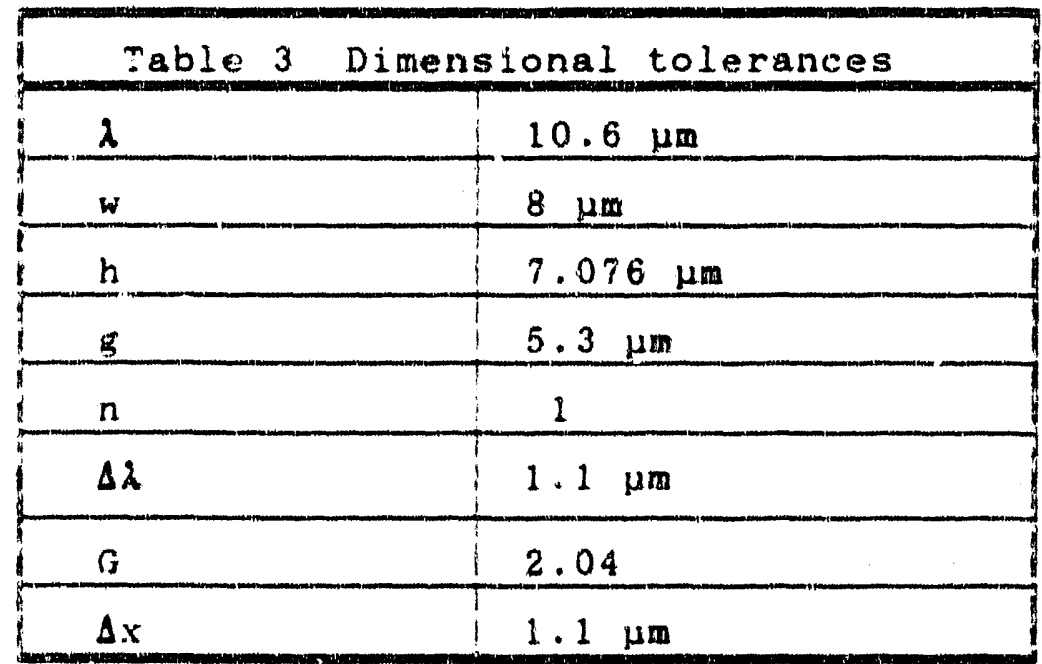

Note that the langer the value of the parameter $G$, the more difficult it is to meet the dimensional tolerance. For this example the contributions to $G$ from $w$ and $h$ are roughly the same.

\section{Time averaged forces}

In terms of particle dynamics the foxhole structure behaves very much like a conventional linac [14]. We discuss the interaction of particles with the fields inside the foxhole to show this. It will be convenient to adopt a new coordinate system with the origin located on the beam axis and centered in the cavity, as shown in Fig. 8. In this coordinate system the standing wave solution of Eq. 13 becomes

$$
\begin{aligned}
& E_{z}=E_{\lambda} \cos \left(\frac{\pi x}{\omega}\right) \cos \left(\frac{\pi y}{h}\right) \cos (\omega t) \\
& B_{x}=\frac{\lambda}{2 h} \frac{E_{\lambda}}{C} \cos \left(\frac{\pi x}{\omega}\right) \sin \left(\frac{\pi y}{h}\right) \sin (\omega t) \\
& B_{y}=-\frac{\lambda}{2 \omega} \frac{E_{\lambda}}{C} \sin \left(\frac{\pi x}{\omega}\right) \cos \left(\frac{\pi y}{h}\right) \sin (\omega t)
\end{aligned}
$$

Consider particle with velocity $\approx c$ and charge $q$ traveling along the $z$ axis. The forces on the particle at a given time are

$$
\begin{aligned}
& F_{x}=-q C B_{y} \\
& F_{y}=q C B_{x} \\
& F_{z}=g D_{z}
\end{aligned}
$$

We can define the phase of a particle by

$$
\text { - } \frac{2 \pi z_{0}}{\lambda}
$$


where $z_{0}$ is the position at $t=0$.

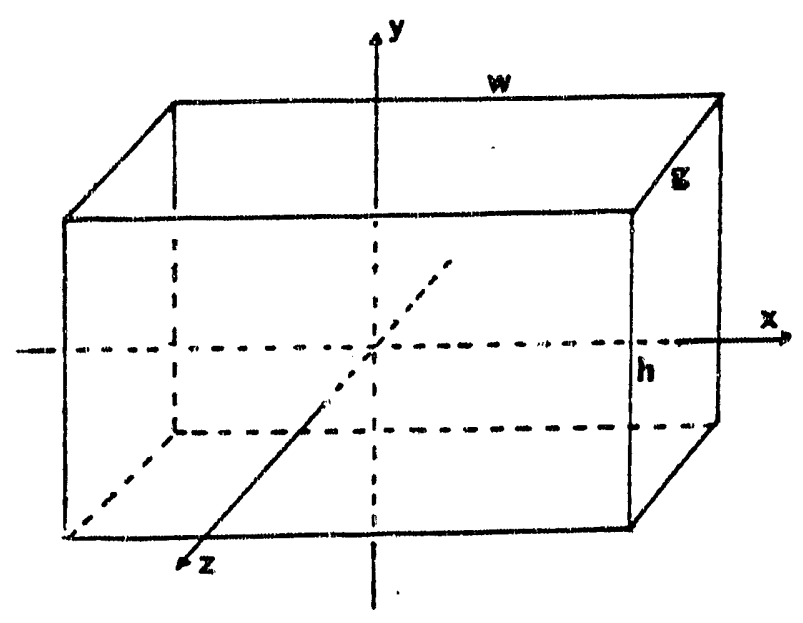

Fig. 8 Coordinate system for beam-cavity interactions.

We consider a foxhole design with gap $g=\lambda / 2$. The limits of the time integration $t_{1}$ to $t_{2}$ are

$$
\begin{aligned}
& t_{1}=-\frac{g}{c}=-\frac{\lambda}{2 c} \\
& t_{2}=+\frac{g}{c}=+\frac{\lambda}{2 c}
\end{aligned}
$$

corresponding to a relativistic particle crossing one spatial period of the structure. For this calculation we assume the fields are identically zero inside the beam channel.

The net force on the particle can then be found by integrating the time dependent force over one period of the structure. For each force component we have

$$
\left\langle F_{1}\right\rangle=\frac{\int_{t_{2}}^{t_{2}} F_{1}(t) d t}{\int_{t_{1}}^{t_{2}} d t}
$$

This quantity is closely related to the transit time factor in conventional linac theory. The integral in the denominator has the 
FOXHOLE STRUCTURE / 26

value $\lambda / c$. We find that

$$
\begin{aligned}
& \left\langle F_{x}\right\rangle=\frac{g \lambda E_{\lambda}}{2 \pi w} \sin \left(\frac{\pi x}{w}\right) \cos \left(\frac{\pi y}{h}\right) \sin (0) \\
& \left\langle F_{y}\right\rangle=\frac{g \lambda E_{\lambda}}{2 \pi h} \cos \left(\frac{\pi x}{w}\right) \sin \left(\frac{\pi y}{h}\right) \sin (1) \\
& \left\langle F_{z}\right\rangle=\frac{q E_{\lambda}}{\pi} \cos \left(\frac{\pi x}{w}\right) \cos \left(\frac{\pi y}{h}\right) \cos (\phi)
\end{aligned}
$$

Note that the transverse forces cancel out exactly for particles moving along the axis $(x=0, y=0)$ and for phases corresponding to the zeros of the sine function. These equations are only valid in the open part of the structure and they do not include any contribution from the fringe field in the vicinity of the opening into the beam ciannel.

We now examine the transverse equations of motion for particles in the vicinity of the beam axis. For $x \approx 0$ and $y \approx 0$ the time averaged force components are

$$
\begin{aligned}
& \left\langle F_{x}\right\rangle=\frac{q \lambda E_{\lambda}}{2 W^{2}} \times \sin (\Phi) \\
& \left\langle F_{y}\right\rangle-\frac{q \lambda E_{\lambda}}{2 h^{2}} y \sin (\Phi) \\
& \left\langle F_{z}\right\rangle-\frac{q E_{\lambda}}{\pi} \cos (\Phi)
\end{aligned}
$$


These components can be equated to the time rate of change of the relativistic momentum. For particles moving with velocity $\approx c$ along the $z$ axis and ignoring the the change in $\gamma$ crossing one gap, we have

$$
\begin{aligned}
F_{x} & =\frac{d}{d t}\left(\gamma m v_{x}\right)=c \frac{d}{d z}\left(m \gamma c \frac{d}{d z} x\right) \\
& =m c^{2} \gamma \frac{d}{d z}\left(\frac{d x}{d z}\right)
\end{aligned}
$$

with is similar equation for $y$. Thus the equations of motion are

$$
\begin{aligned}
& m c^{2} \gamma \frac{d}{d z}\left(\frac{d x}{d z}\right)=\frac{q \lambda E_{\lambda}}{2 w^{2}} \sin (\Phi) x \\
& m c^{2} \gamma \frac{d}{d z}\left(\frac{d y}{d z}\right)=\frac{q \lambda E_{\lambda}}{2 h^{2}} \sin (\Phi) y
\end{aligned}
$$

The equations of motion are similar to those for conventional quadrupoles

$$
x^{\prime \prime}-k_{z} x=0
$$

with a similar equation for $y$, where

$$
\begin{aligned}
& k_{X}=\frac{1}{m c^{2} \gamma} \frac{q \lambda E_{A}}{2 W^{2}} \sin (\Phi) \\
& k_{Y}=\frac{1}{m c^{2} \gamma} \frac{q \lambda E_{A}}{2 h^{2}} \sin (\Phi)
\end{aligned}
$$

Numerically, for $50 \mathrm{MeV}$ electrons with $E_{\Lambda}=1 \mathrm{GV} / \mathrm{m}, \lambda=10 \mu \mathrm{m}$, and foxhole dimensions on the order of half a wavelength, we have

$$
k_{x, y} \sim 10^{7} \sin (\Phi) \quad\left[m^{-2}\right]
$$

Note that $k_{q}$ and $k_{p}$ have the same sign, determined by $\phi$. For $\Phi<0$ there is transverse focussing and longitudinal defocussing, while for $\Phi>0$ there is transverse defocussing and longitudinal focussing.

\section{MAFIA model}

The analytic calculations given up to this point have disregarded the presence of channels between successive holes for the electron beam to pass through. We studied the effects caused by such 
channels numerically by means of the 3D EM field code MAFIA [10]. This approach has its own limitations which must be taken into account in the interpretation of the results. They stem from the nature of the code and from the constraints on available memory and speed of the computer used. The essential result of a MAFIA run is a set of field values at all mesh points of a finite, three dimensional mesh at a number of successive discrete instants in time. These values may deviate from equivalent ones obtained analytically because MAFIA substitutes repeated evaluation of finite difference equations at each meshpoint and its immediate neighbours in space and time for integration of differential equations. This may be acceptable provided that the fields, which are continuous in space and time, have negligible structure on the scale of the inesh size, both in space and in time. This is certain to be the case if the mesh size is small compared to the structure in the boundaries, because it is that which determines the structure in the fields. It also limits the size of the problems that can be fruitfully explored in a mesh of prescribed size, particularly if the boundary conditions are geometrically complex. Sudden changes in the boundary conditions, such as sharp edges at the intersections between metallic plane surfaces, violate the requirement of smooth change and can cause problems. The edges between the foxhole and the top surface, and between the foxhole and the electron beam channel are examples.

To maximize the resolution available with a fixed number of mesh points one exploits the symmetry properties of the problem at hand. of the whole foxhole we modeled only one half period along $z$ (see Fig. 9), consisting of one half-foxhole and one half-channel, bounding the problem space by two flat surfaces, perpendicular to the system axis, at $z=1$ and at $z=\frac{1}{2} \beta \lambda$. The tangential components of $E$ and the normal component of $B$ are required to be zero in these planes, or, more precisely, at the mesh points located in these planes. Then we made use about the left-right symmetry about the system axis, bounding the problem space by a plane through that axis and perpendicular to the $x$ direction. The normal component of $E$ and the tangential components of $B$ are required to be zero in this plane. The base of the problem space is the horizontal plane coplanar with the bottom of the foxhole. We require the tangential components of $E$ to be zero in this plane.

The two remairing boundaries of the problem space (perpendicular to the positive $x$ and $y$ axes) lie at infinity for the true foxhole structure. This is not realizable with a finite number of mesh points. One possibility would be to enforce a radiation boundary, i.e., a surface on which the ratio between the perpendicular components of $E$ and $H$ is kept fixed at the impedance of free space, $Z_{0}$, but the version of MAFIA available to us does not have that feature. We therefore enclosed the problem space with a plane at large $x$ and another one at large $y$, imposing the requirement that the normal component of $E$ and the tangential components of $B$ vanish on both. This changes the problem to be solved from that of a plane 
wave, traveliing in open space, reflected and scattered by the foxhole structure, into that of the eigenmodes of a closed cavity. Where originally the reflected and scattered radiation disappears into space, leaving the incident radiation unperturbed, it is now retained and changes the distribution of the incident radiation at the orifice of the foxhole in a way that is not easily predictable.

A physical realization of the MAFIA model would consist of a baseplate with not one foxhole structure, but an infinite periodic array of parallel foxhole structures, faced by its mirror image in a plane of symmetry, rather than by free space. While there are many modes, only those that resonate with the lowest mode of the foxhole proper are of interest. The frequency of that mode is only approximately known, because of the uncertainty caused by the boundary shape at the orifice. We put the offending boundaries at several wavelengths from the mouth of the foxhole, as indicated in Table 4 , in order to increase the mode density in the vicinity of the foxhole mode. Doing so requires additional mesh points and reduces the resolution available in the foxhole itself. All the following MAFIA calculations were done with a uniformly spaced mesh.

The behaviour in the time domain is similarly complex. Since in the model there is no dissipation and since no radiation can leave the cavity, the $Q^{\prime}$ s of the individual modes are all very (infinitely) large and their bandwidths correspondingly narrow (zero). All modes, except the ones that are coupled, will continue to oscillate as independent oscillators with energies that are determined by the initial conditions. Different modes can only be coupled if they have identical resonant frequencies. It is practically impossible to excite only the desired modes, because the initial conditions needed to do that are determined by the unknown mode structure. It would require correct specification of the fields at all inesh points at a particular instant.

Similar information as generated by a MAFIA model could be obtained by measurements in a physical model. The concerns discussed above would not be applicable, or only with a much reduced extent. They would be replaced by others, primarily about sensitivity and noise of the measuring equipment. The linearity of Maxwell's equations permit linear scaling of the model to the convenience of the experimenter. Dissipation would be unavoidable and could be selectively controlled to some extent; it would be possible to provide a good approximation of a radiation boundary. The physical correctness of the results can not be doubted, although, of course, their interpretation can be. However, this approach requires a laboratory, electronic equipment, design, construction and 
FOXHOLE STRUCTURE / 30

\begin{tabular}{|l|l|}
\hline Table 4 MAFIA model of foxhole structure \\
\hline Mesh points & \\
\hline $\mathrm{x}$ & 36 \\
\hline $\mathrm{y}$ & $44-61$ \\
\hline $\mathrm{z}$ & 21 \\
\hline$\lambda$ & 10 \\
\hline $\mathrm{w}$ & 10 \\
\hline $\mathrm{h}$ & 5.75 \\
\hline $\mathrm{g}$ & 5 \\
\hline (4 mesh points per unit problem interval) \\
\hline
\end{tabular}

subsequent modifications of the model and much time, while numerical models require only an office, a computer terminal and also time. Although there is little doubt that our effort will ultimately require physical model work, we derided that the numerical work described below would help to improve our understanding of the foxhole structure.

\section{2 slotted channel structure}

Any actual accelerating structure must have a channel or tunnel for the beam to pass through. This channel must be big enough to encompass the tails of the particle transverse distribution, yet small enough so that it does not significantly disturb the field patterns in the cavity. The slotted structure is easier to build than a buried channel. It can be constructed by etching down along the $y$ direction into a single piece of silicon. On the other hand, since the slot extends the full height of the cavity, it causes a greater disturbance in the cavity fields. Fig. 9 shows a MAFIA model of a slotted channel structure. 


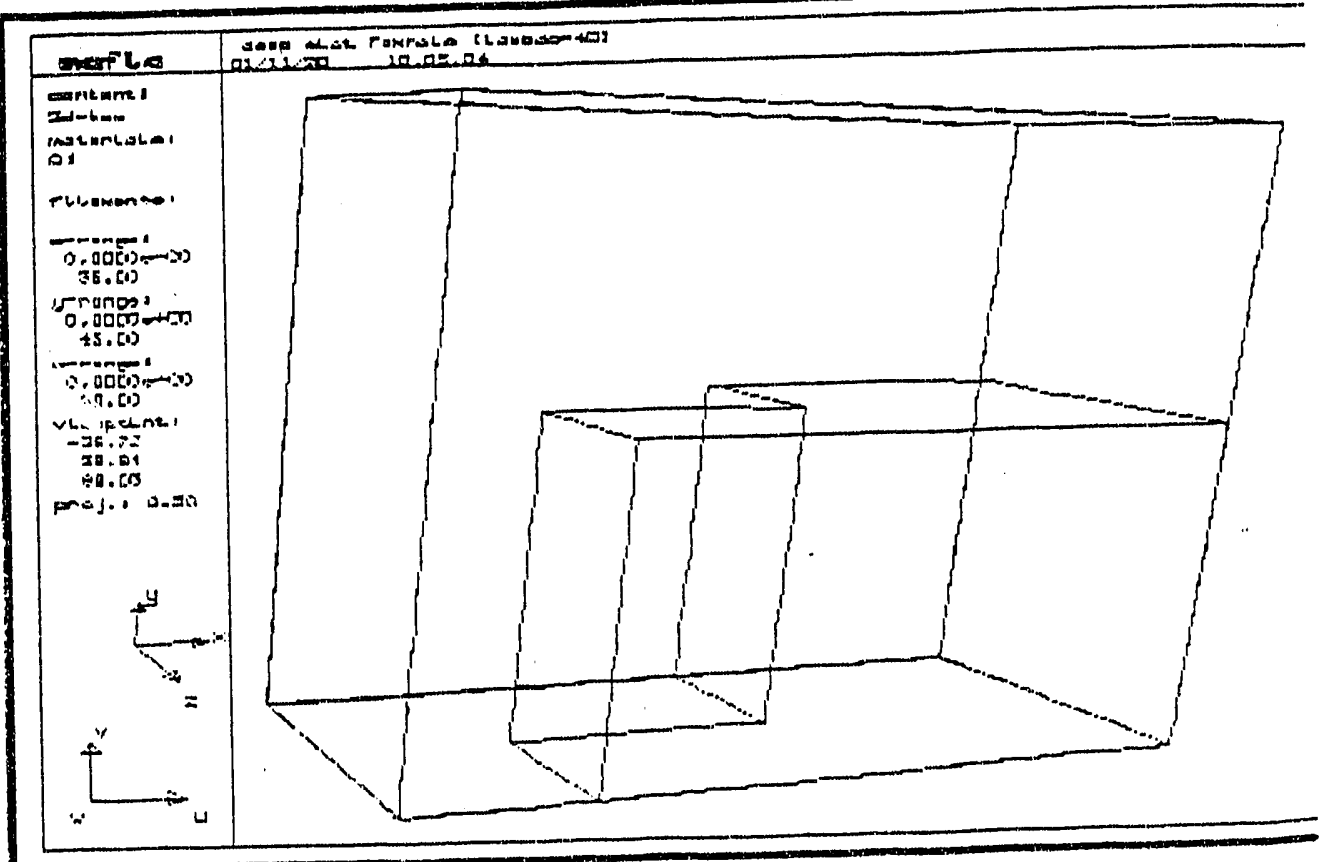

Fig. 9 MAFIA model of the slotted channel foxhole structure. The electron travels along the $z$ axis and the radiation is incident along the $-y$ axis.

Fig. 10 shows the ratio of the accelerating field in the cavity to the field in the space above the cavity as a function of $s$, the total width of the slot. All other dimensions in the problem were kept fixed. The quantity that is actually computed is

$$
\frac{E_{A}}{E_{0}}=\frac{\int_{0}^{\frac{g}{2}} E_{z}\left(0, h / 2, z, t_{0}\right) d z}{\int_{0}^{\frac{g}{2}} E_{z}\left(0,3 h / 2, z, t_{0}\right) d z}
$$

The integrals are called "deflection integrals" in MAFIA. They are useful for computing a spatial average of the field at a fixed time. The relative field in the cavity falls off quickly for increasing slot width.

The calculations became less reliable as $s$ increased. This is 
FOXHOLE STRUCTURE / 32

indicated by the parameter $f_{y}$ in Fig. 10 , which gives the ratio of

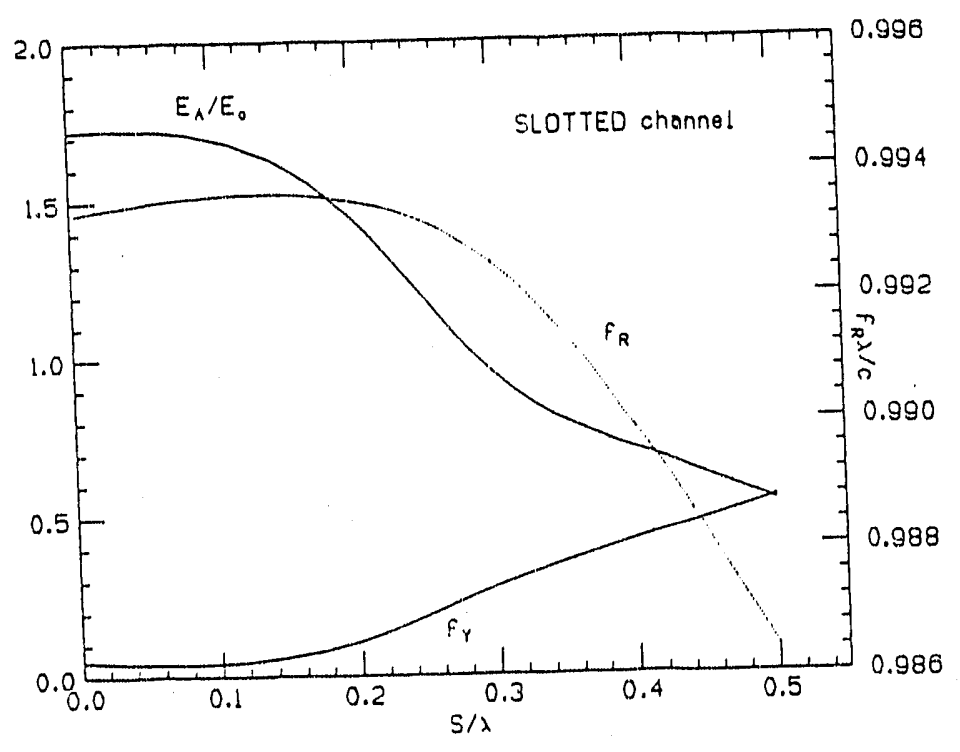

Fig. 10 Left axis: The ratio of the accelerating field to the incident field as a function of the slot width. Also shown is the relative magnitude of the $y$ component of the electric field in the space above the structure. Right axis : Resonant frequency of the structure.

the $y$ to the $z$ component of the electric field in the space above the cavity:

$$
f_{Y}=\frac{\int_{0}^{\frac{g}{2}} E_{Y}\left(0,3 h / 2, z, t_{0}\right) d z}{\int_{0}^{\frac{g}{2}} E_{z}\left(0,3 h / 2, z, t_{0}\right) d z}
$$

We have assumed that the space above the foxhole contains a standing wave electromagnetic field along $y$. This field should not have any component of the electric field along $y$. If it does, our MAFIA model is not a true representation of a foxhole. Fig. 10 indicates that the program results are suspect for large values of s. This may result from the restricted total number of mesh points $(\approx 50,000)$.

The dependence of the resonance frequency of the cavity on $s$ is also shown in Fig. 10. The frequency is rather flat for $s$ up to $0.2 \lambda$, but falls of $f$ rapidly for greater $s$. Note that for a particle 
traversing the cavity in phase with the resonating field, the scale on the right side of Fig. 10 also gives the relativisitic velocity factor

$$
\beta=\frac{V_{P}}{C}=\frac{\omega_{R}}{c k}=\frac{f_{R} \lambda}{C}
$$

for the particles that move synchronously through the accelerating structure.

We next consider MAFIA calculations of the integlated forces on a particle travelling parallel to the $z$ axis with velocity $c$ and crossing a half cell of the structure (cavity plus channel). The calculated forces take into account the time variation of the fields. The $F_{2}$ force is shown in Fig. 11 as a function of $x_{p}$, the $x$ position of a particle crossing the foxhole. The particles cross the cavity with $y_{p}$ equal to half the cavity height. All particles travel with the phase that gives maximum acceleration. The slot widths are expressed in any system of length where $\lambda=10$ units (in other words $s=2$ means $s / \lambda=0.2$ ). We see that $F_{2}$ drops rapidly as the slot width is increased, but it is relatively insensitive to the $x_{p}$ position for non-zero slot dimensions.

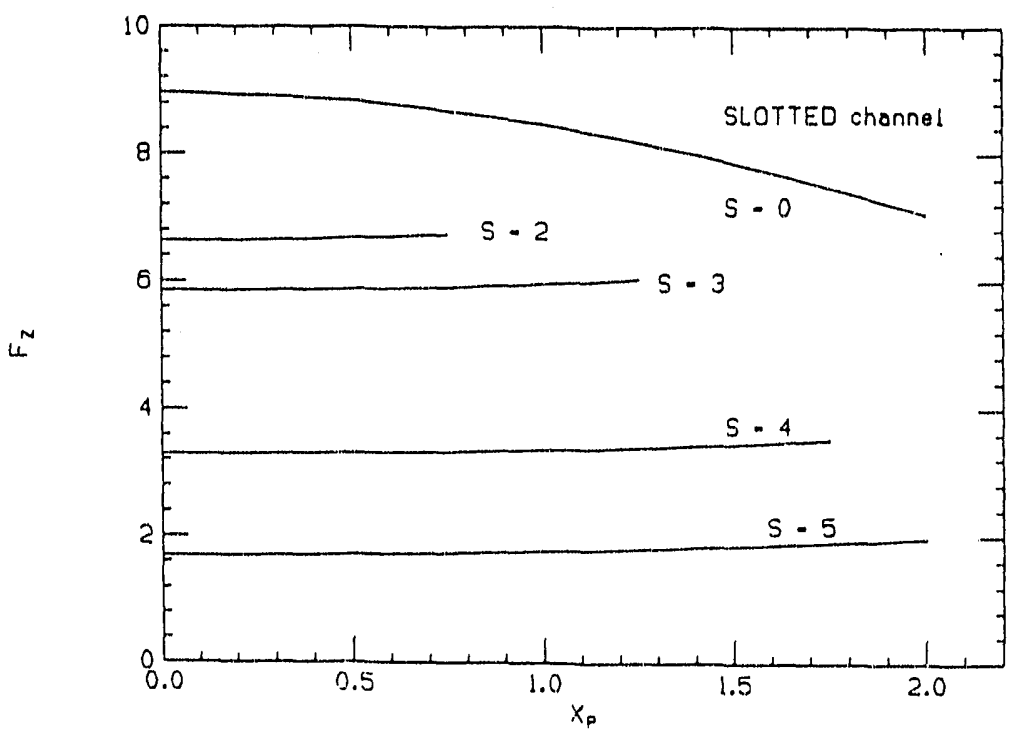

Fig. 11 The integrated accelerating force $F_{7}$ in volts as a function of the transverse position $x_{p}$ of a particle. The curves refer to various slot widths $\left(\lambda=10, y_{p}=\right.$ $\mathrm{h} / 2)$.

Fig. 12 shows that the slot causes an $x$ directed force that tends to become less significant for larger slot widths. Recall from Eq. 68 that the transverse forces are $90^{\circ}$ out of phase with respect to 
$F_{\eta}$. Thus there is no contribution to the transverse forces from the cavity fields in this case. This was verified in a MAFIA run with a slot width of zero. The transverse force contribution in Fig. 12 and in the following figures is due to the fringe field in the vicinity of the slot edges. The calculated MAFIA value $F_{y}$ is actually a potential expressed in volts. It is related to the analytic, time-averaged force in $\mathrm{Eq} .67$ by

$$
F_{x}^{*}-\frac{F_{x} \lambda}{q}
$$

where $A$ is the slope of the curve in Fig. 12. We can relate this to the quadrupole strength parameter of $\mathrm{Eq} .72$ by

$$
k_{x} \sim \frac{q A}{m c^{2} \gamma \lambda}
$$

Using $A \approx 1 \mathrm{~V} / \mu \mathrm{m}, E=50 \mathrm{MeV}$, and $\lambda=10 \mu \mathrm{m}$, we find that $\mathrm{k}_{\mathrm{y}}$ from the channel fringe fields is $\approx 2000 / \mathrm{m}^{2}$. This is small compared with the equivalent quadrupole strengths due to the transverse cavity forces for a finite length bunch.

Fig. 13 shows the variation in $F_{p}$ as a function of $x_{p}$. These transverse forces were calculated using the phase for maximum acceleration. Note that the magnitude of $F_{k}$ is about a factor of 5 smaller and $F_{p}$ is about a factor of 30 smaller than the magnitude of $F_{\eta}$.

The dependence of the integrated force $F_{q}$ on the deviation of $y_{p}$ from the half-height of the cavity is shown in Fig. 14. The particles cross the cavity with $x_{p}=0 . F_{z}$ has a significant dependence on $y_{p}$. Unless it is corrected, this effect would cause a correlation to develop between the momentum of particles in a beam bunch and their y position. 


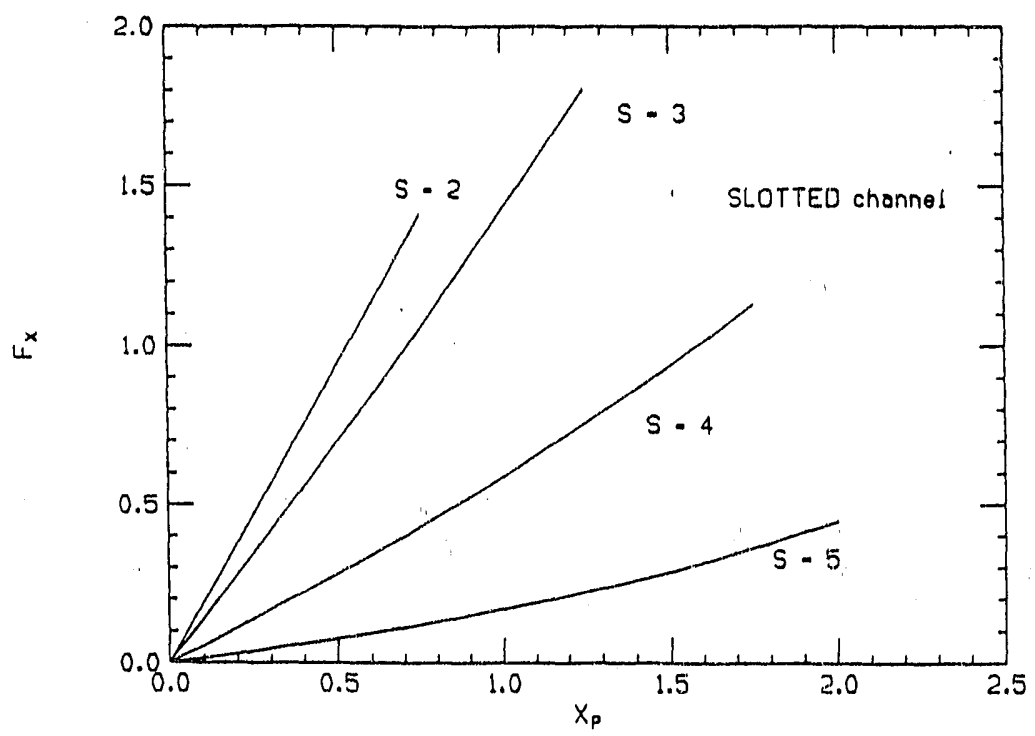

Fig. 12 The integrated deflecting force $F_{z}$ in volts as a function of the transverse position $x_{p}$ of a particle. The phase corresponds to maximum acceleration. The curves refer to various slot widths $\left(\lambda=10, y_{p}=h / 2\right)$.

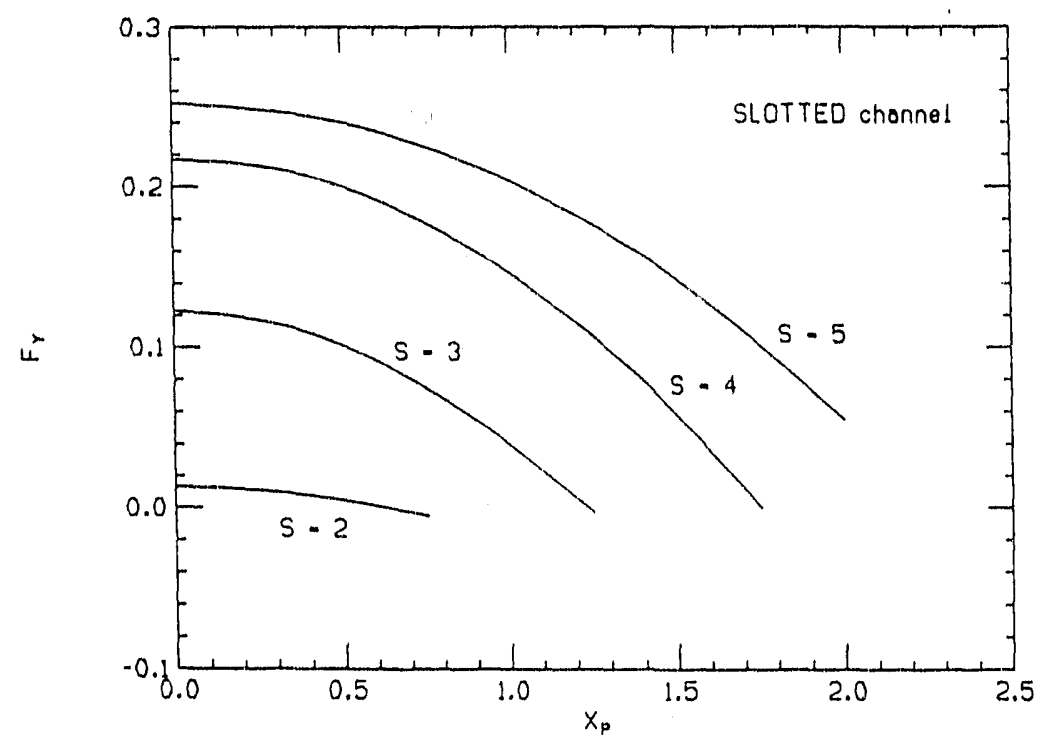

Fig. 13 The integrated deflecting force $F_{9}$ in volts as a function of the transverse position $x_{p}$ of a particle. The phase corresponds to maximum acceleration. The curves refer to various slot widths $\left(\lambda=10, y_{p}=h / 2\right)$. 
FOXHOLE STRUCTURE / 36

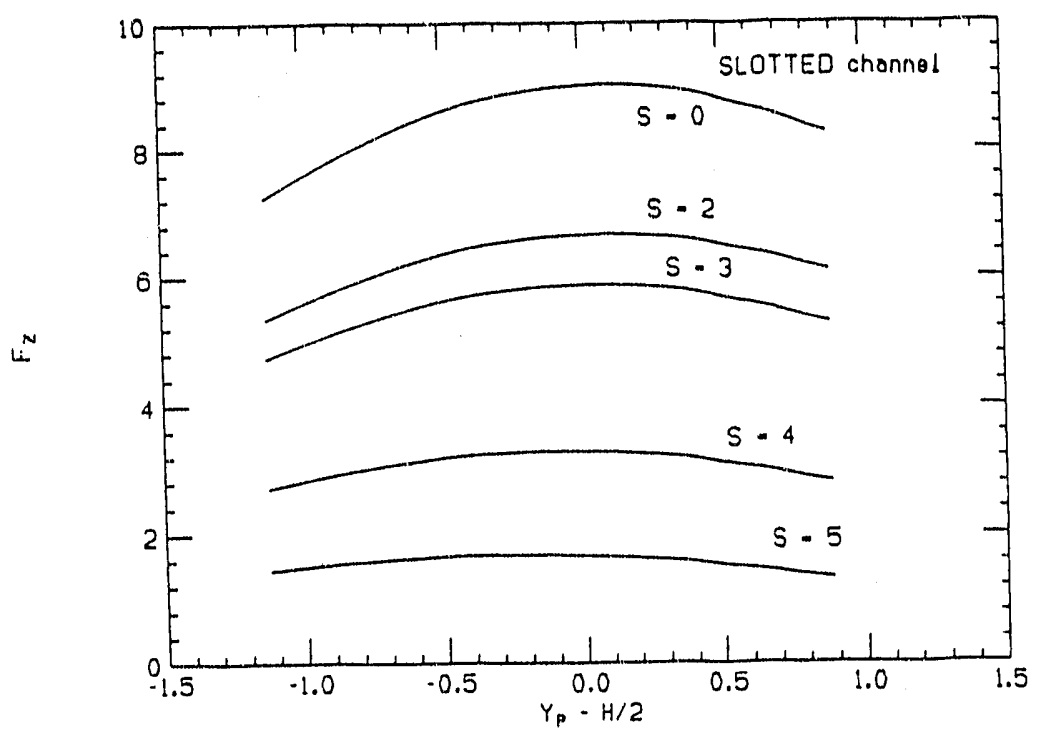

Fig. 14 The integrated accelerating force $F_{\eta}$ in volts as a function of the transverse position $y_{p}$ of a particle. The curves refer to various slot widths $\left(\lambda=10, x_{p}=0\right)$.

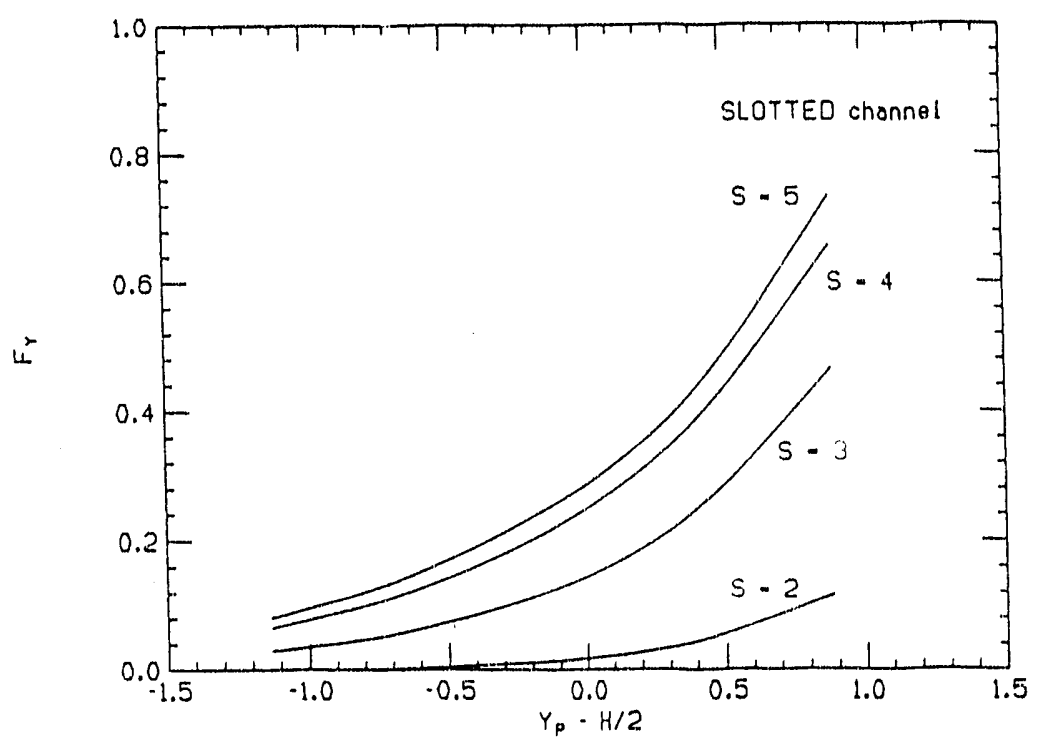

Fig. 15 The integrated deflecting force $F_{p}$ in volts as a function of the transverse position $y_{p}$ of a particle. The phase corresponds to maximum acceleration. The curves refer to various slot widths $\left(\lambda=10, x_{p}=0\right)$. 
Fig. 15 shows that all particles in the slot see the same sign vertical force. Thus there is no axis with $F_{y}=0$, and $F_{y}$ is asymmetric for particles above and below the beam axis. Particles near the open surface of the foxhole experience the largest vertical forces, particularly for large slot widths. Because of symmetry there is no dependence of $F_{x}$ on $y_{p}$ for $x_{p}=0$.

\section{Buried channel structure}

It is more difficult to manufacture a buried channel structure than a slotted channel one. It requires etching along the $x$ direction into two halves of the structure and then precisely aligning and joining the pieces together. Fig. 16 shows a MAFIA model of a buried channel structure. We use a square channel for the calculations.

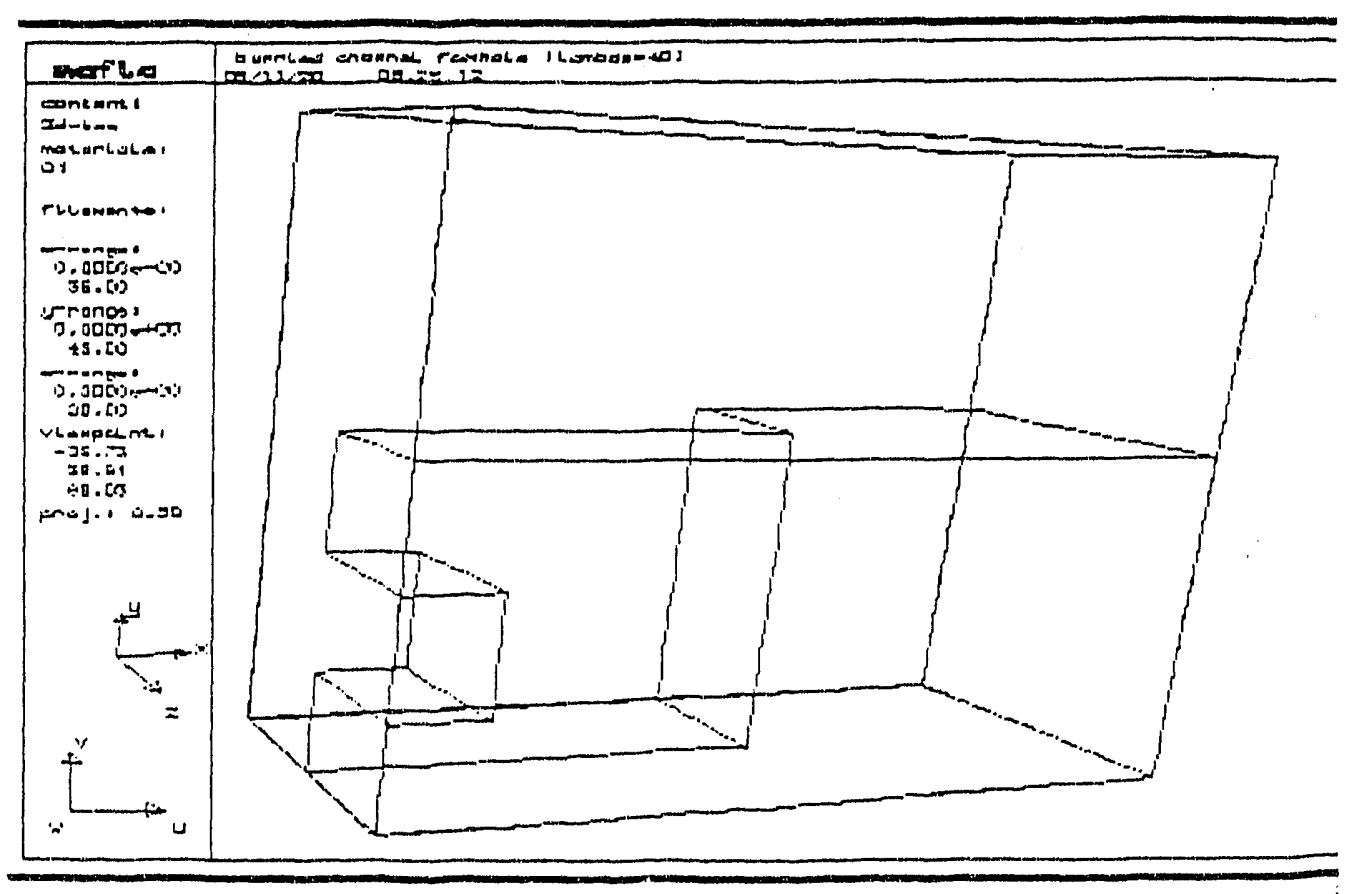

Fig. 16 MAFIA model of the buried channel foxhole structure; $\mathbf{s}$ : the total width of the slot. The electron travels along the $z$ axis and the radiation is incident along the $-y$ axis.

Fig. 17 shows the ratio of the integrated $E_{z}$ field in the cavity to the $E_{\mathrm{g}}$ field in the space above the cavity as a function of $s$, the total width of the channel. The peak accelerating field falls of $f$ with increasing $s$ roughly the same as for the slotted channel. Again the parameter $f_{3}$ shows that the calculations become less 
reliable at large $s$. Fig. 17 also shows a roughly linear increase in the resonance frequency as $s / \lambda$ is increased from 0.2 to 0.4 .

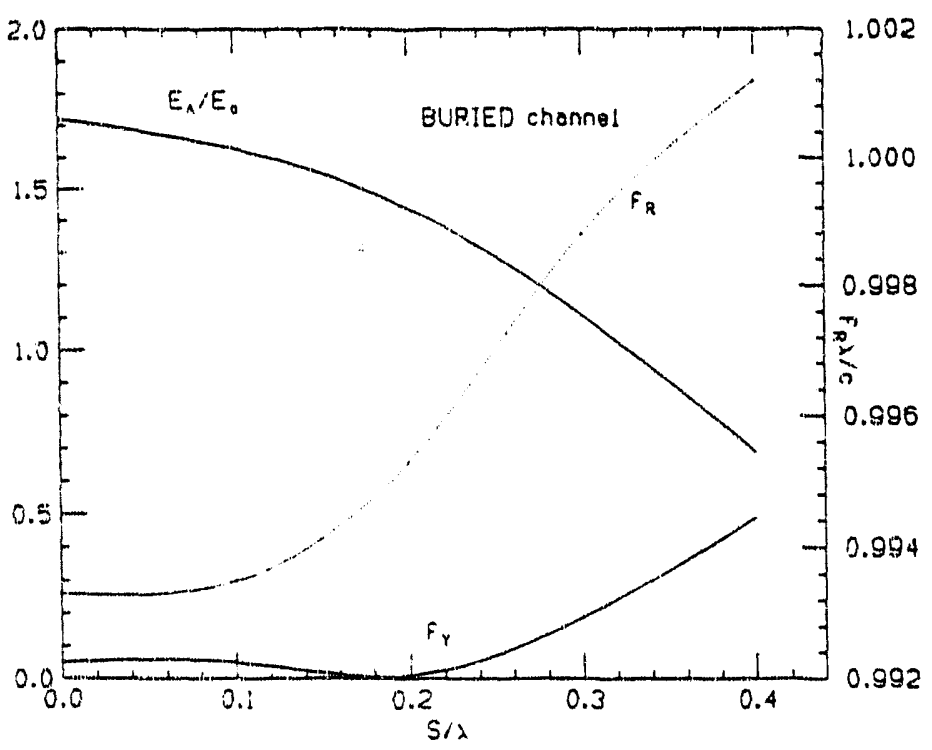

Fig. 17 Left axis: The ratio of the accelerating field to the incident field as a function of the slot width. Also shown is the relative magnitude of the $y$ component of the field in the srace above the structure.

Right axis: Resonant frequency of the structure.

MAFIA calculations of the integrated forces on a particle crossing a half cell of the structure (cavity plus channel) is shown in Fig. 18 as a function of $x_{n}$, the $x$ position of a particle crossing the foxhole. The particles cross the cavity parallel to the $z$ axis with velocity $c$ and with $y$ equal to half the cavity height. All particles travel with the phase that gives maximum acceleration. Fig. 18 shows that the integrated value of $F$ drops off rapidy with increasing channel width and is practically independent of $x$ for channel widths less than 4. Comparing Fig. 18 with Fig. Il shows that the net force in the $z$ direction is significantly higher for the buried channel, despite the fact that the peak accelerating field in the open cavity is roughly the same for the two cases. This would seem to indicate that the field inside the channel contributes more effectively for the buried channel.

Fig. 19 shows that the channel causes an $x$ directed force that grows with $x_{y}$. There is no tendency for $F_{x}$ to decrease and flatten out for large $s$, as there is with the slotted channel. The dependence of $F$, on $x_{n}$ is inuch weaker than for the slotted channel; the magnitude did not exceed 0.04 for the cases studied. 


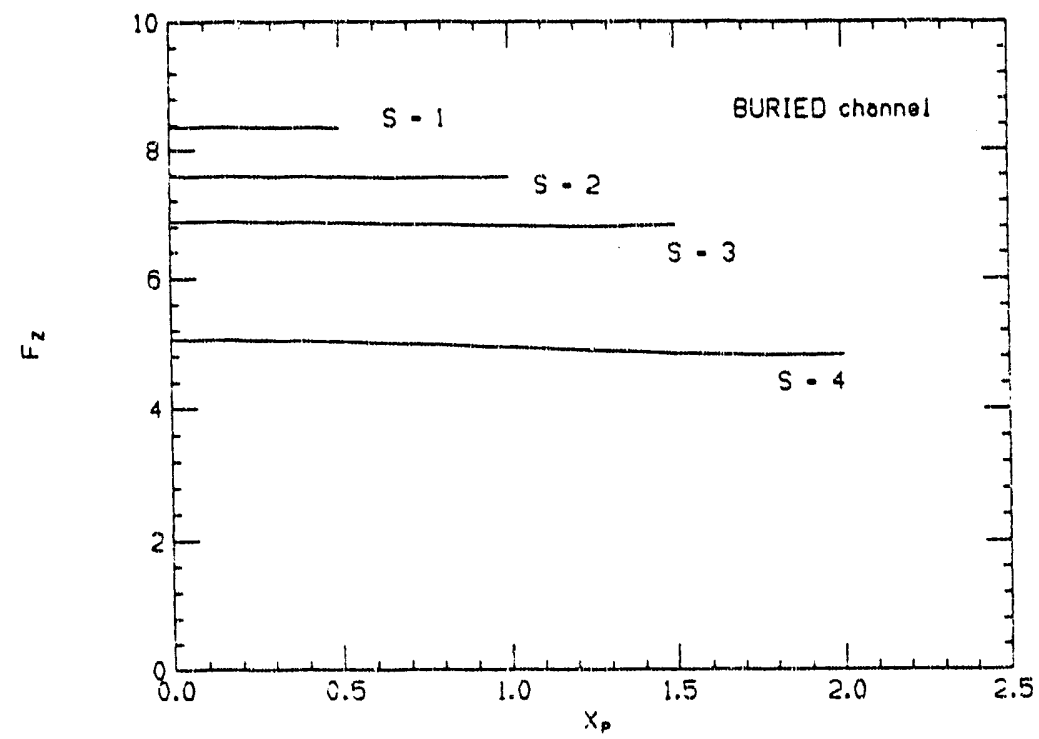

Fig. 18 The integrated accelerating force $F_{y}$ in volts as a function of the transverse position $x_{p}$ of a particle. The curves refer to various slot widths $\left(\lambda=10, y_{p}=\right.$ h/2).

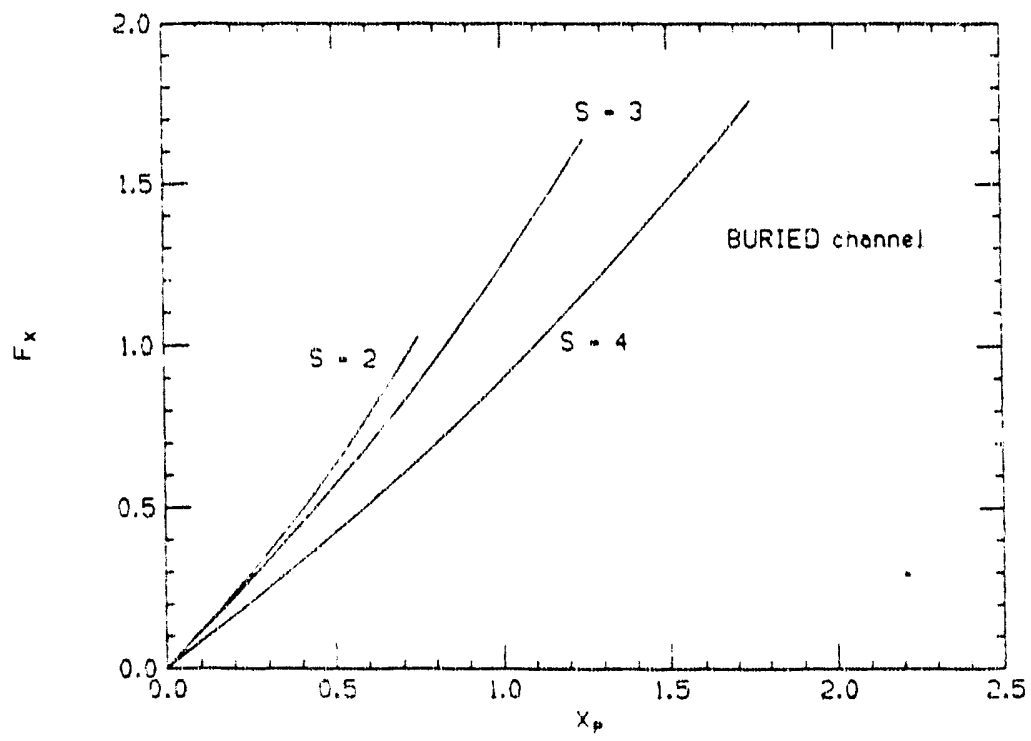

Fig. 19 The integrated deflecting force $F_{y}$ in volts as a function of the transverse position $x_{p}$ of a particle. The phase corresponds to maximum acceleration. The curves refer to various slot widths $\left(\lambda=10, y_{p}=h / 2\right)$. 
The dependence of the integrated force on the deviation of $y$ from the half-height of the cavity is shown in Fig. 20. The particles cross the cavity with $x_{p}=0$. $F_{q}$ has a significant dependence on $y_{p}$. Unless it is corrected, this effect would cause a correlation to develop between the momentum of particles in a beam bunch and their y position.

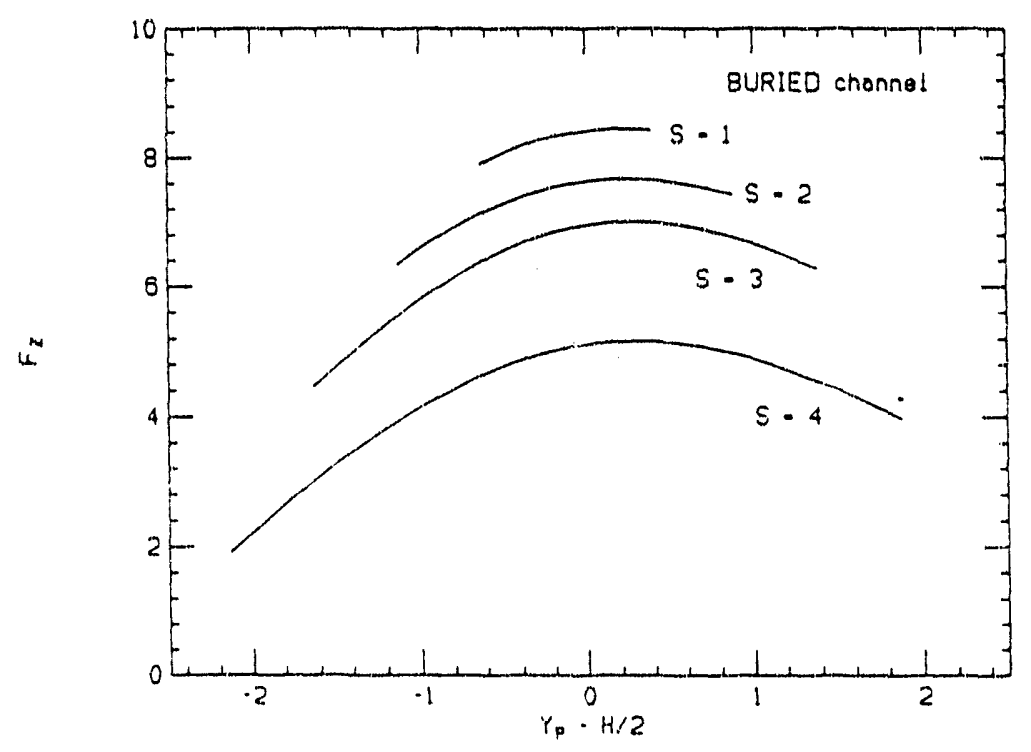

Fig. 20 The integrated accelerating force $F_{7}$ in volts as a function of the transverse position $y_{p}$ of a particle. The curves refer to various slot widths $\left(\lambda=10, x_{p}=0\right)$.

Fig. 21 shows that, unlike the slotted structure, the buried channel structure has no net $F$ on the beam axis. In this respect the forces more closely follow the ideal behavior described by Eqs. 69.

We have also studied the MAFIA model shown in Fig. 22, which encorporates a full cell along $z$. Fig. 23 shows the electric field pattern in a plane that slices vertically through the center of the structure. One can see the accelerating electric field lines inside the open cavity and the field lines decreasing inside the channel. There is a node in the field near the top of the cavity and a plane wave-like field in the space above it. Fig. 24 shows a horizontal slice at the half height of the cavity. The electron beam axis is on the right edge of the figure. 


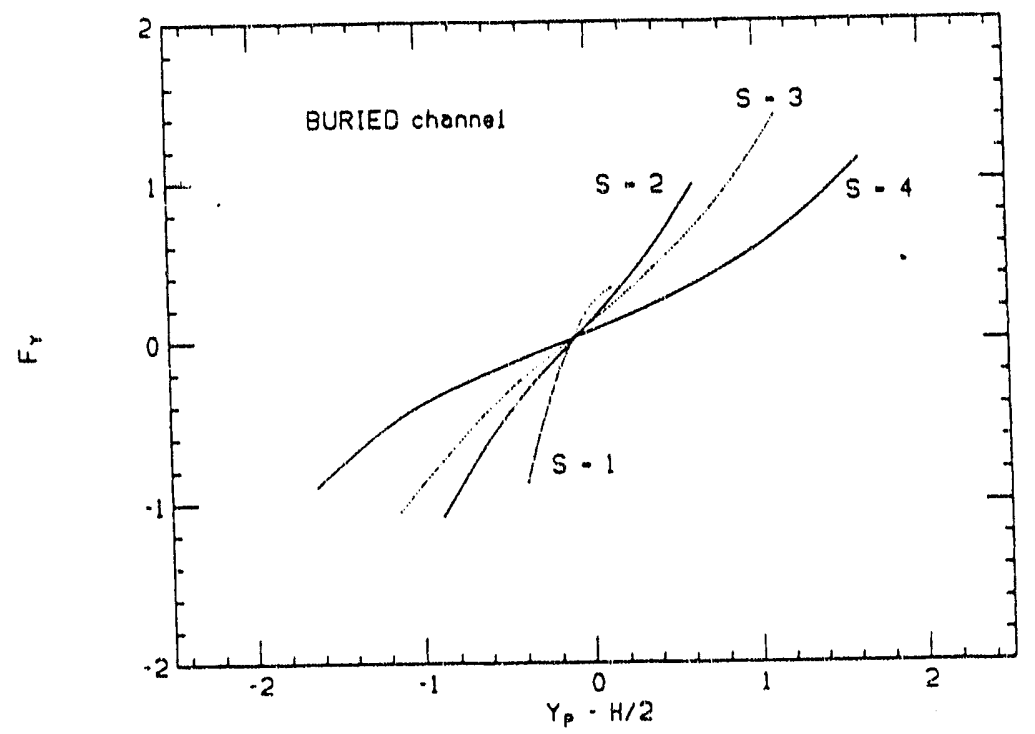

Fig. 21 The integrated deflecting force $F_{\text {p }}$ in volts as a function of the transverse position $y_{p}$ of a particle. The phase corresponds to maximum acceleration. The curves refer to various slot widths $\left(\lambda=10, x_{p}=0\right)$.

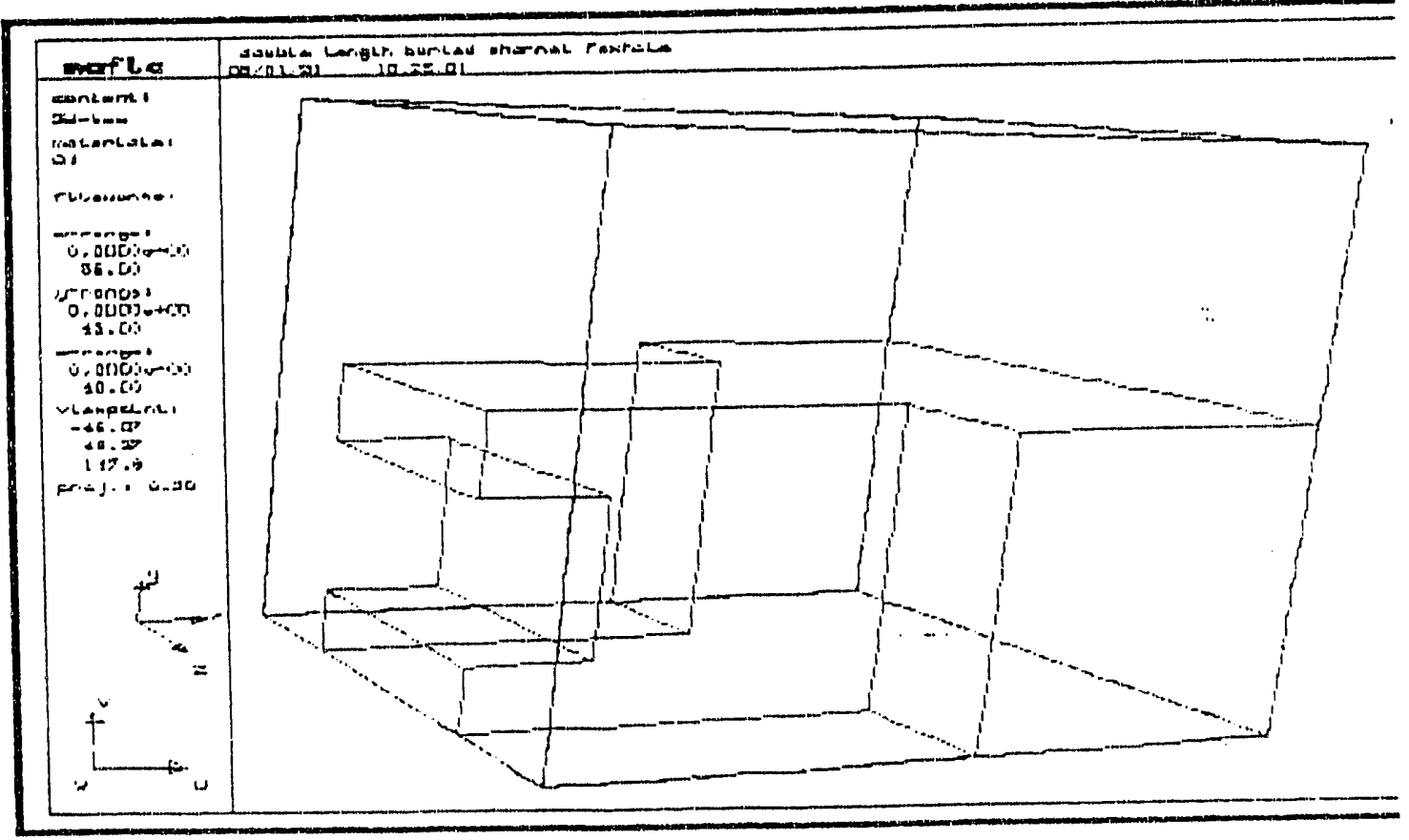

Fig. 22 MAFIA model of a full longitudinal cell of the buried channel, foxhole structure. Because of symmetry only half of the cell is modelled in the transverse $(x)$ direction. 


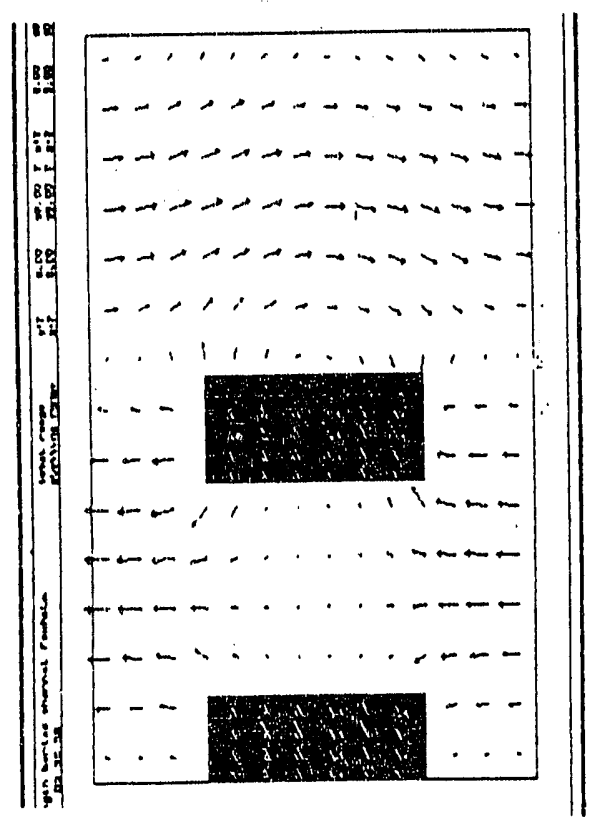

Fig. 23 MAFIA results for the electric field vectors in a vertical slice through the center of the buried channel, foxhole structure.

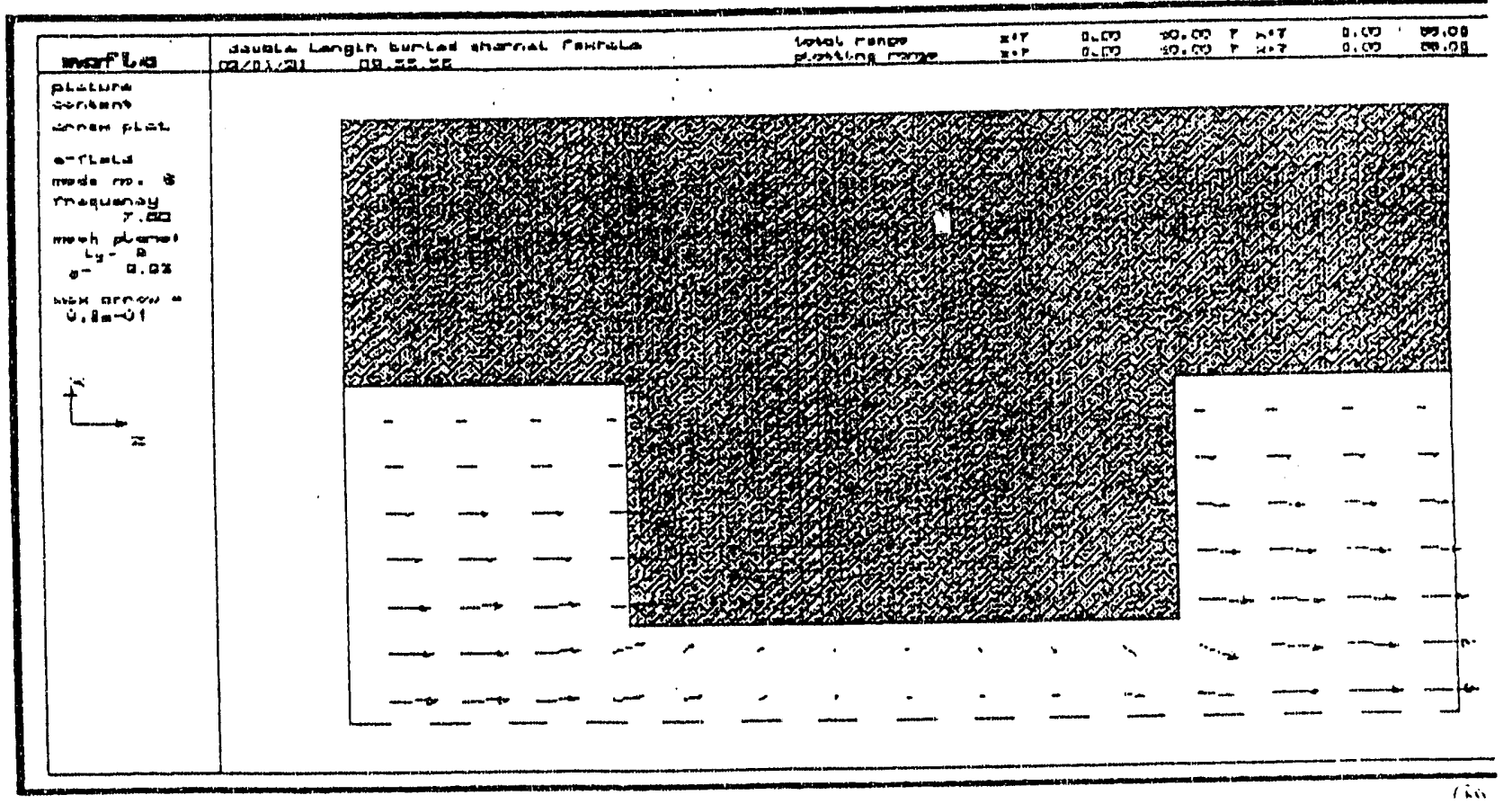

Fig. 24 MAFIA results for the electric field vectors in a horizontal slice through the center of the buried channel, foxhole structure. 


\section{Design dimensions}

The first demonstration of the performance of a foxhole cavity could take place in the Grating Acceleration experiment[11] now under construction at the Accelerator Test Facility(ATF)[12] at Brookhaven National Laboratory. The radiation source is a high power, short pulse carbon dioxide laser. We will summarize the conclusions of the preceeding sections by presenting reference designs for a slotted channel and a buried channel structure.

The ATF will deliver a beam to the accelerating structure with an rms transverse radius of $0.7 \mathrm{\mu m}$. In order to accept most of the beam the foxhole must have a slot width of at least $3 \mu \mathrm{m}$. Since we have seen that the presence of the slot causes a shift in the resonance frequency of the cavity, we compute the quantity

$$
\Delta E=\frac{f(s=3)-f(s=0)}{f(s=0)}
$$

We can find the sensitivity of the frequency shift to the cavity height by taking the differential of Eq. 15. We find that

$$
\frac{d h}{d \xi}=\frac{-4 h^{3}}{n^{2} \lambda^{3}}
$$

Using this expression we can compute the change in height $\Delta$ h that

\begin{tabular}{|c|c|c|}
\hline Table 5 & Slotted cha & design \\
\hline$g$ & 5.3 & $\mu \mathrm{m}$ \\
\hline$w$ & 8 & $\mu \mathrm{m}$ \\
\hline $\mathrm{n}$ & 1 & \\
\hline$h_{0}$ & 7.076 & $\mu \mathrm{m}$ \\
\hline$s$ & 3 & $\mu \mathrm{m}$ \\
\hline$s / \lambda$ & 0.283 & \\
\hline$\Delta \xi$ & $-5.03 \times 10^{-4}$ & \\
\hline$\partial \mathrm{h} / \partial \xi$ & -12.61 & $\mu \mathrm{m}$ \\
\hline$\Delta h$ & 0.006 & $\mu \mathrm{m}$ \\
\hline
\end{tabular}
is required to bring the cavity back on resonance.

The required height change for the slotted channel is much smaller than the dimensional tolerance of $\approx 1 \mu \mathrm{m}$ calculated earlier and can be ignored. 


\begin{tabular}{|l|l|l|}
\hline \multicolumn{2}{|c|}{ Table 6} & Buried channel design \\
\hline$g$ & 5.3 & $\mu \mathrm{m}$ \\
\hline$w$ & 8 & $\mu \mathrm{m}$ \\
\hline $\mathrm{n}$ & 1 & \\
\hline$h_{0}$ & 7.076 & $\mu \mathrm{m}$ \\
\hline$s$ & 3 & $\mu \mathrm{m}$ \\
\hline$s / \lambda$ & 0.283 & \\
\hline$\Delta \xi$ & $4.93 \times 10^{-3}$ & \\
\hline$\partial \mathrm{h} / \partial \xi$ & -12.61 & $\mu \mathrm{m}$ \\
\hline$\Delta \mathrm{h}$ & -0.062 & $\mu \mathrm{m}$ \\
\hline
\end{tabular}

Although the required dimensional change is larger for this case, it is still much smaller than the allowed dimensional tolerance and can be neglected.

\section{Conclusions}

We have used simple analytic arguments and approximate computer modeliing to show that the foxhole structure should be suitable for accelerating relativistic electrons. Reasonable geometries should give accelerating fields several times greater than the peak magnitude of the incident radiation. The technology exists now to make slotted channel structures that couple to optical radiation. Unfortunately the slotted channel. foxhole has a relatively large, asymmetric vertical force that would have to be compensated. The buried channel foxhole on the other hand has a neutral axis where both transverse forces vanish. In this respect it acts similarly to the conventional disk-loaded structure.

Although we have discussed this structure in the context of laser driven acceleration, it is of course not restricted to use at optical wavelengths. It may be a reasonable choice for any wavelength between $10 \mu \mathrm{m}$ and $1 \mathrm{~cm}$, where building a conventional, closed cavity would be difficult or impossible. 
Notes \& References

[1] See for example the review, R.H. Siemann, Advanced accelerator concepts and electron-positron linear colliders, Ann.Rev.Nucl.Part.Sci. 37:243-66, 1987, and the references mentioned there.

[2] R.B. Palmer, The interdependence of parameters for TeV linear colliders, in $S$. Turner(ed), New Developments in Particle Acceleration Techniques, p. 80-121, CERN Report 87-11, 1987.

[3] J. Claus, Energy efficiency and choice of parameters for linear colliders, in M. Puglisi et al(eds), New Techniques for Future Accelerators, p. 45-65, Ettore Majorana International Science Series 2g, Physical Sciences, Plenum Press, New York, 1987.

[4] P.J. Channel(ed), Laser Acceleration of Particles, AIP Conf. Proc. No. 91, 1982; C. Joshi \& T. Katsouleas(eds), Laser Acceleration of Particles, AIP Conf. Proc. No. $130,1985$.

[5] R.B. Palmer, Open accelerating structures, in S. Turner(ed), op.cit., p. 633-41; N. Kroll, General features of the accelerating modes of open structures, in C. Joshi \& T. Katsouleas(eds), op.cit., p. 253-70.

[6] J. Claus, Characteristics of foxhole resonator, unpublished notes, 18 June 1986; J. Claus, Laser linac: foxhole structure, unpublished notes, Jan. 1990.

[7] J.D. Jackson, Classical Electrodynamics, Wiley, 1962.

[8] The minus sign in the first Eq. 7 comes from the fact that the wave is traveling in the $-y$ direction. The derivative with respect to $y$ is then negative.

[9] P. Lorrain \& D. Corson, Electromagnetic Fields and Waves, 2nd ed., Freeman, 1970, p. 560-1.

[10] MAFIA Users Guide, February 23, 1987.

[11] W. Chs l et al, Proposal for a study of laser acceleration using microstrating structures at the ATF, BNL report $43465,1989$.

[12] K. Batchelor et al, Operational status of the Brookhaven National Laboratory Accelerator Test Facility, Proc. 1989 Particle Accelerator Conference, p. 273.

[13] Taken from R. Harrington, Time-harmonic Electromagnetic Fields, McGraw-Hill, 1961, p. 75 . 
FOXHOLE STRUCTURE / 46

[14] See for example, L. Smith, Linear accelerators, in S. Flugge (ed,), Handbuch der Physik, Vol. 44, Springer-Verlag, Berlin, 1959 , p. $344-7$. 

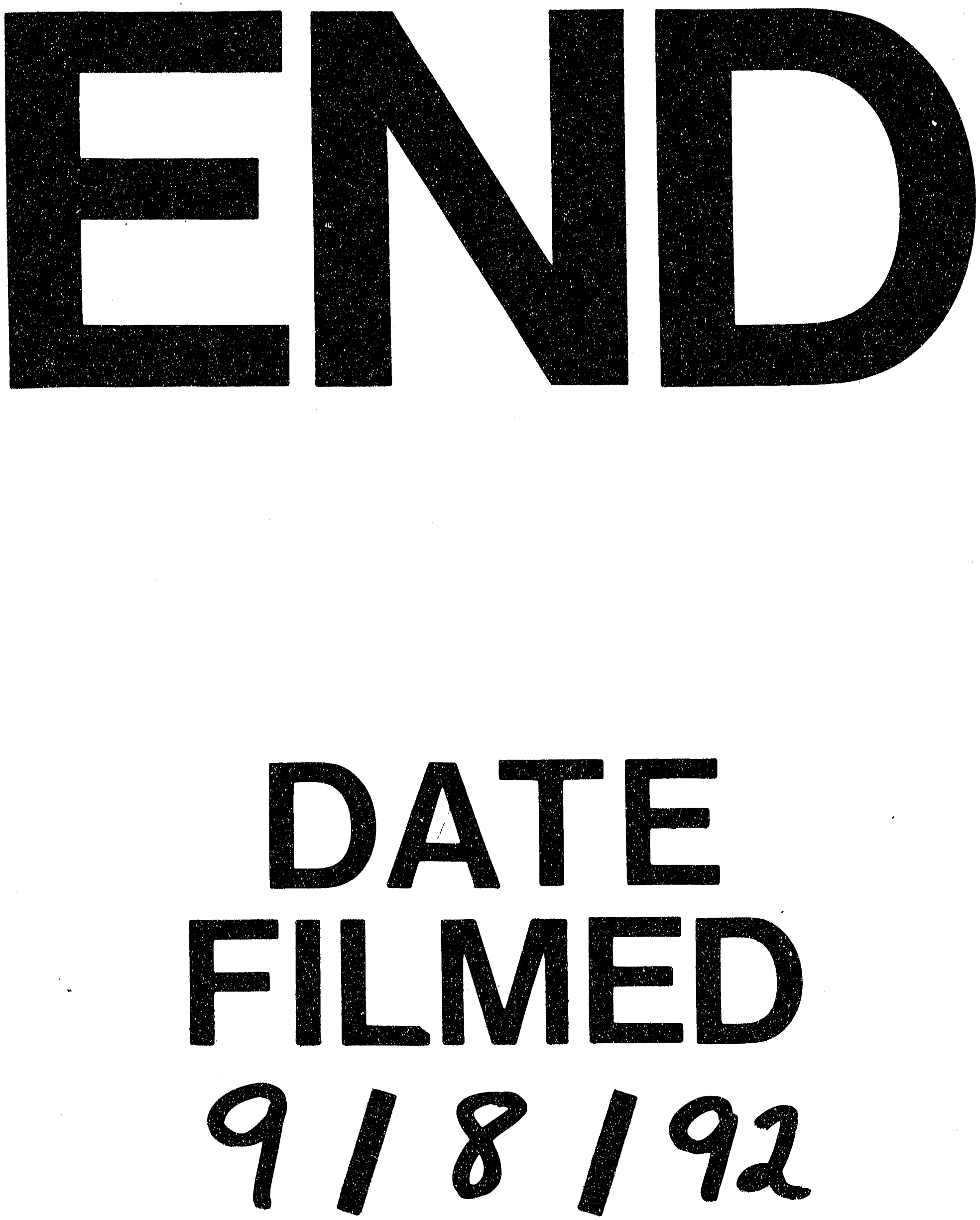
